\title{
Periodic points for piecewise monotonic transformations
}

\author{
FRANZ HOFBAUER \\ Institut für Mathematik, Universität Wien, Strudlhofgasse 4, A-1090 Wien, Austria
}

(Received 4 May 1983 and revised 12 January 1984)

Abstract. Periodic points of piecewise monotonic transformations are investigated using an oriented graph, whose closed paths represent the periodic orbits of the transformation. In the first part it is shown that the inverse of the $\zeta$-function is a kind of characteristic polynomial of this graph, which is a power series if the graph is infinite. In the second part, the sets are determined, which can occur as $\{n \in \mathbb{N}: \exists x$ with $T^{n}(x)=x$ and $T^{i}(x) \neq x$ for $\left.0<i<n\right\}$, where $T$ is a monotonic $\bmod$ one transformation.

\section{Introduction}

The aim of this paper is the investigation of periodic points of piecewise monotonic transformations $([0,1], T)$. One calls $T$ piecewise monotonic if $[0,1]=\bigcup_{i=1}^{N} J_{i}$, where the $J_{i}$ are non-trivial disjoint intervals and $T \mid J_{i}$ is continuous and monotone. Sometimes it is more appropriate to consider the shift space $\left(\Sigma_{T}^{+}, \sigma\right)$ one gets from $([0,1], T)$ by $f$-expansion, especially if $([0,1], T)$ has infinitely many periodic points of a fixed period (cf. lemma 1 of $\S 1$ ).

The method we use is that of an oriented graph, called a Markov diagram, which reflects the orbit structure of $\Sigma_{T}^{+}$. The first part of the paper investigates the $\zeta$-function of $\Sigma_{T}^{+}$given by

$$
D(x)=\exp \left(\sum_{k=1}^{\infty} \frac{x^{k}}{k} p_{k}\right),
$$

where $p_{k}$ denotes the number of fixed points of $\sigma^{k}: \Sigma_{T}^{+} \rightarrow \Sigma_{T}^{+}$. In $\S 2$ we define a characteristic power series $C(x)$ of the Markov diagram considered as 0 -1-matrix, which is an analogy to the characteristic polynomial of a matrix. We then show that $C(x)^{-1}$ essentially equals the $\zeta$-function. From this it follows that the kneading invariant, defined in [8] for the special case of continuous piecewise monotonic transformations, is the characteristic power series of the Markov diagram. This sheds light on the fact that both methods, the Markov diagram and the kneading invariant, have been used to determine the topological structure of piecewise monotonic transformations. In the first case the Markov diagram is split up into irreducible submatrices (cf. [2]-[6]) and in the second case the kneading invariant, i.e. the characteristic power series of the Markov diagram, is split up into the corresponding product (cf. [7]).

Then we turn to the investigation of the set

$$
Z(T)=\left\{n: \exists x \in[0,1] \text { with } T^{n}(x)=x \text { and } T^{i}(x) \neq x \text { for } 1 \leq i \leq n-1\right\} .
$$


For continuous $T$, the theorem of Šarkovskǐi (cf. [1]) gives a nice characterization of all possible sets $Z(T)$. For general piecewise monotonic transformations $T$ many more sets can occur as $Z(T)$, as we shall see at the end of $\S 2$. In $\S 3$ we investigate monotonic mod 1 transformations $T$ on $[0,1)$, i.e. there is a continuous increasing function $h:[0,1) \rightarrow \mathbb{R}$ such that $T(x)=h(x) \bmod 1$. If we consider 1 as a periodic point, if $\lim _{t \uparrow \uparrow} T^{k}(1)=1$ for some $k$, and if $L$ is a maximal topologically transitive subset of $([0,1], T)$, we get the following result for $Z(T \mid L)$ :

There are integers $p, q$ and real numbers $\lambda, \mu$ with $0 \leq \lambda \leq 1 \leq \mu \leq \infty$ such that $\{v p+w q: \lambda<w / v<\mu$ and $v, w \in \mathbb{N}\} \subset Z(T \mid L) \subset\{v p+w q: \lambda \leq w / v \leq \mu$ and $v, w \in \mathbb{N}\}$. We also determine

$$
Z(T \mid L) \cap\{v p+w q: w / v=\alpha \text { and } v, w \in \mathbb{N}\} \quad \text { for } \alpha=\lambda \text { and } \alpha=\mu,
$$

(cf. theorem 2). From this one can deduce which sets can occur as $Z(T)$ for monotonic mod 1 transformations $T$ (cf. theorem 3).

\section{Preliminaries}

Let $([0,1], T)$ be piecewise monotonic and let $J_{i}$ for $1 \leq i \leq N$ be the intervals on which $T$ is monotone and continuous. The f-expansion $\varphi:[0,1] \rightarrow\{1, \ldots, N\}^{\mathbb{N}}$ is defined by

$$
\varphi(x)=\underline{x}=x_{0} x_{1} x_{2}, \ldots, \quad \text { where } x_{i} \text { is such that } T^{i}(x) \in J_{x_{i}} .
$$

One sees easily that $\sigma \circ \varphi=\varphi \circ T$. We define

$$
\Sigma_{T}^{+}=\overline{\varphi([0,1])}
$$

If $u$ and $v$ are the left and right endpoints of $J_{i}$ respectively, we set

$$
\underline{a}^{i}=\lim _{t \downarrow u} \varphi(t) \quad \text { and } \quad \underline{b}^{i}=\lim _{t \uparrow v} \varphi(t) .
$$

A characterization of $\Sigma_{T}^{+}$in terms of $\underline{a}^{i}$ and $\underline{b}^{i}$ is given in [2]. If

$$
Y=\bigcup_{k=0}^{\infty} \sigma^{-k}\left(\left\{\underline{a}^{i}, \underline{b}^{i}: 1 \leq i \leq N\right\}\right),
$$

then $\Sigma_{T}^{+} \backslash \varphi([0,1]) \subset Y$ (cf. [2]). For $\underline{x} \in \varphi([0,1])$, one easily checks that $\varphi^{-1}(\{\underline{x}\})$ is either a single point or an interval. We compare the periodic points of $\left(\Sigma_{T}^{+}, \sigma\right)$ and of $([0,1], T)$. We say an $x \in[0,1]$ has period $n$ if $T^{n}(x)=x$ and $T^{i}(x) \neq x$ for $1 \leq i \leq n-1$. We use the same definition for $\left(\Sigma_{T}^{+}, \sigma\right)$. The proof of the following lemma is easy.

LEMMA 1. If $\underline{x} \in \Sigma_{T}^{+}$is not periodic, then $\varphi^{-1}(\{\underline{x}\})$ contains no periodic point.

$Y$ contains at most finitely many periodic points. Suppose $\underline{y} \in \Sigma_{T}^{+} \backslash Y$ has period $n$.

(i) If $\varphi^{-1}(\{y\})$ is a single point $y \in[0,1]$, then $y$ has period $n$.

(ii) If $\varphi^{-1}(\{\underline{y}\})$ is an interval $I$, then $T^{n}(I) \subset I$ and $I$ contains at least one periodic point. If $T^{n} \mid I$ is increasing, all these periodic points in $I$ have period $n$. If $T^{n} \mid I$ is decreasing, one of them has period $n$ and all others have period $2 n$.

Lemma 1 shows the following: to investigate $\left(\Sigma_{T}^{+}, \sigma\right)$ instead of $([0,1], T)$ means to consider equivalence classes of periodic points, where $x$ and $y \in[0,1]$ are called 
equivalent if $\varphi(x)=\varphi(y)$, and to add finitely many periodic points, which are in $Y$. For the investigation of $\left(\Sigma_{T}^{+}, \sigma\right)$ we use the Markov diagram, which we define now.

The Markov diagram is a finite or countable oriented graph, whose vertices are subsets of $\Sigma_{T}^{+}$. Set $\left[x_{0} x_{1} \cdots x_{k-1}\right]=\left\{y \in \Sigma_{T}^{+}: y_{i}=x_{i}\right.$ for $\left.0 \leq i \leq k-1\right\}$. If $D \subset[i]$ for some $i$ with $1 \leq i \leq N$, we call the non-empty sets among $[j] \cap \sigma(D)$ for $1 \leq j \leq N$ the successors of $D$. Let $\mathscr{D}$ be the set which contains $[i]$ for $1 \leq i \leq N$, and which contains all successors of $D$ if it contains $D$. To get the oriented graph, which we call Markov diagram, we insert an arrow from every $D \in \mathscr{D}$ to all its successors. We write $D \rightarrow C$ to denote that $C$ is a successor of $D$.

A sequence $D_{0} D_{1} D_{2} \cdots$ with $D_{i} \in \mathscr{D}$ is called a path if $D_{i} \rightarrow D_{i+1}$. Remark that all $D \in \mathscr{D}$ satisfy $D \subset[i]$ for some $i$. Hence we can define $\psi(D)=i$, if $D \subset[i]$. We say that the path $D_{0} D_{1} D_{2} \cdots$ represents $\underline{x}$, if $\underline{x}=\psi\left(D_{0}\right) \psi\left(D_{1}\right) \psi\left(D_{2}\right) \cdots$. The following two important facts about the representation of points $x \in \Sigma_{T}^{+}$are proved in [5]: If $D \in \mathscr{D}$, then

$D=\left\{\underline{x} \in \Sigma_{T}^{+}: \underline{x}=\psi\left(D_{0}\right) \psi\left(D_{1}\right) \cdots\right.$ for some path $D_{0} D_{1} \cdots$ with $\left.D_{0}=D\right\}$

If $\underline{x} \in \Sigma_{T}^{+} \backslash Z$, where $Z=\left\{\sigma^{k}\left(\underline{a}^{i}\right), \sigma^{k}\left(\underline{b}^{i}\right): 1 \leq i \leq N, k \geq 0\right\}$, and $\underline{x}$ is represented by the paths $C_{0} C_{1} C_{2} \cdots$ and $D_{0} D_{1} D_{2} \cdots$, then:

$$
\text { there is a } t \in \mathbb{N} \text { with } C_{i}=D_{i} \text { for } i \geq t \text {. }
$$

We call an ordered $n$-tuple $D_{0} D_{1} \cdots D_{n-1}$ with $D_{i} \in \mathscr{D}$ a closed path of length $n$ if we have $D_{i} \rightarrow D_{i+1}$ for $0 \leq i \leq n-2$ and $D_{n-1} \rightarrow D_{0}$, and if there is no divisor $m$ of $n$ with $D_{i}=D_{i+k m}$ for $0 \leq i \leq m-1$ and $1 \leq k \leq(n / m)-1$. Such a path represents the periodic point $\underline{x}=\psi\left(D_{0}\right) \psi\left(D_{1}\right) \cdots \psi\left(D_{n-1}\right) \psi\left(D_{0}\right) \psi\left(D_{1}\right) \cdots$. The properties (1.4) and (1.5) have the following consequence. Let $x \in \Sigma_{T}^{+}$be of period $n$. As $\underline{x} \in\left[x_{0}\right] \in \mathscr{D}, \underline{x}$ is represented by a path $D_{0} D_{1} D_{2} \cdots$ with $D_{0}=\left[x_{0}\right]$, by (1.4). Since $\sigma^{n}(\underline{x})=\underline{x}, \underline{x}$ is also represented by the path $D_{n} D_{n+1} D_{n+2} \cdots$. If $\underline{x} \notin Z$, there is a $t$ with $D_{n+i}=D_{i}$ for $i \geq t$, by (1.5). Hence $x$ is represented by the closed path $D_{s} D_{s+1} \cdots D_{s+n-1}$, where $s=j n \geq t$, which has length $n$. If it had length $<n$, it would follow that $D_{i}=D_{i+r}$ for all $i \geq s$ and some $r<n$, and $x=\psi\left(D_{s}\right) \psi\left(D_{s+1}\right) \cdots$ would have period $r<n$. It follows also from (1.5) that the representation of $x \notin Z$ by a closed path is unique. For $x \in Z$ and piecewise increasing $T$, the same result except for the uniqueness of the closed path representing $\underline{x}$ is shown in the proof of lemma 7 in [5]. Such an $x$ has finitely many representations as a closed path (cf. lemma 4 below). By the methods of part II of [2], this last result for $\underline{x} \in Z$ holds also in the general case, but the length of the closed path can also be $2 n$. We summarize this in the following lemma.

LEMMA 2. (i) Every $\underline{x} \in \Sigma_{\Gamma}^{+} \backslash Z$ of period $n$ is uniquely represented by a closed path in the Markov diagram, which has length $n$.

(ii) If $\underline{x} \in Z$ has period $n$, then $\underline{x}$ is represented by a closed path finitely many times. These closed paths have length $n$, if $T$ is piecewise increasing. If $T$ is decreasing on some $J_{i}$, some of them can have length $2 n$.

(iii) A point represented by a closed path is periodic. 
Remark. Using (1.4) and (1.5), it is shown in [5] that there is a 1-1 correspondence between maximal topological transitive subsets $\Omega$ of $\Sigma_{T}^{+}$containing a periodic point and maximal irreducible subsets $\mathscr{D}^{\prime}$ of $\mathscr{D}$. The results of [5] imply that lemma 2 holds also for $\Omega$ instead of $\Sigma_{T}^{+}$and $\mathscr{D}^{\prime}$ instead of $\mathscr{D}$.

\section{2. $\zeta$-functions}

In this section we consider the Markov diagram as a $\mathscr{D} \times \mathscr{D}$-matrix $M$ with entries 0 and 1 . For $C, D \in \mathscr{D}$ we define $M_{C D}=1$, if and only if $C \rightarrow D$. Since every $C \in \mathscr{D}$ has at most $N$ successors, $u \mapsto u M$ is an $l^{1}(\mathscr{D})$-operator, whose spectral radius $r(M)$ satisfies $r(M) \leq\|M\|_{1} \leq N$. It is shown in [2] that $\exp h_{\text {top }}\left(\Sigma_{T}^{+}\right) \leq r(M)$. Let $p_{k}$ denote the number of fixed points of $\sigma^{k}: \Sigma_{T}^{+} \rightarrow \Sigma_{T}^{+}$. The $\zeta$-function is defined by

$$
D(z)=\exp \left(\sum_{k=1}^{\infty} \frac{z^{k}}{k} p_{k}\right) \text {. }
$$

This power series converges for $|z|<\exp \left(-h_{\text {top }}\left(\Sigma_{T}^{+}\right)\right)$and hence for $|z|<1 / r(M)$, since $p_{k} \leq \operatorname{card}\left\{\left[x_{0} \cdots x_{k-1}\right]:\left[x_{0} \cdots x_{k-1}\right] \neq \varnothing\right\}$. If $Z$ contains no periodic point, we have by lemma 2 that $p_{k}=\operatorname{tr} M^{k}$, which denotes the trace of the matrix $M^{k}$. Instead of $D(z)$ we consider

$$
\tilde{D}(z)=\exp \left(\sum_{k=1}^{\infty} \frac{z^{k}}{k} \operatorname{tr} M^{k}\right) .
$$

To add a periodic point of period $n$ to $\Sigma_{T}^{+}$means to multiply $D(z)$ by $\left(1-z^{n}\right)^{-1}$. Since each of the finitely many periodic points in $Z$ is represented by finitely many closed paths (cf. lemma 2), we get $D(z)=\tilde{D}(z) \cdot H(z)$, where $H$ and $H^{-1}$ are holomorphic on $\{z:|z|<1\}$. Our goal is to define a characteristic power series $C(z)$ of $M$ and to show $C(z)=\tilde{D}(z)^{-1}$.

To this end set $\mathscr{D}_{1}=\{[i]: 1 \leq i \leq N\} \subset \mathscr{D}$. If $\mathscr{D}_{k} \subset \mathscr{D}$ is defined, let $\mathscr{D}_{k+1}$ be the set which contains $\mathscr{D}_{k}$ and all successors of elements of $\mathscr{D}_{k}$. We have $\mathscr{D}_{k} \subset \mathscr{D}_{k+1}$ and $\bigcup_{k=1}^{\infty} \mathscr{D}_{k}=\mathscr{D}$ by the definition of $\mathscr{D}$. As every $D \in \mathscr{D}$ has at most $N$ successors, the sets $\mathscr{D}_{k}$ are finite. Set $M_{k}=M \mid \mathscr{D}_{k}$. We call a closed path $D_{0} D_{1} \cdots D_{n-1}$ self-avoiding if $D_{i} \neq D_{j}$ for $0 \leq i<j \leq n-1$. For $L=M_{k}$ or $L=M$ let $b_{n}^{i}(L)$ be the number of $i$-tuples of self-avoiding closed paths in $L$, which are pairwise disjoint and the sum of whose lengths is $n$. Set $a_{0}(L)=1$ and for $n \geq 1$ set $a_{n}(L)=\sum_{i=1}^{n}(-1)^{i} b_{n}^{i}(L)$. The following lemma is easy to prove.

LeMma 3. For the characteristic polynomial $C_{k}(z)=\operatorname{det}\left(I-z M_{k}\right)$ of $M_{k}, I$ the unit matrix, one has

$$
C_{k}(z)=\sum_{n \geq 0} a_{n}\left(M_{k}\right) z^{n}
$$

We define the characteristic power series of $M$ by $C(z)=\sum_{n=0}^{\infty} a_{n}(M) z^{n}$ which is justified by theorem 1 , for which we need the following lemma.

LEMMA 4. (i) There is a finite or empty set of closed paths in the Markov diagram, each of which represents an $\underline{x} \in Z$, such that a closed path of length $n$, which is not in this set, is contained in $\mathscr{D}_{2 n-1}$. If $T$ is piecewise increasing, it is even contained in $\mathscr{D}_{n-1}$. 
(ii) There is a $k_{0}$ such that $k \geq k_{0}$ and $k \geq 2 n-1$ imply $b_{n}^{i}\left(M_{k}\right)=b_{n}^{i}(M), a_{n}\left(M_{k}\right)=$ $a_{n}(M)$ and $\operatorname{tr} M_{k}^{n}=\operatorname{tr} M^{n}$.

Proof. We show (i) only if $T$ is piecewise increasing. From this one easily gets the general result using the method of part II of [2].

It is shown by lemma 12 of [2], that $\mathscr{D}$ can be written as

$$
\mathscr{D}=\{(A, i, k),(B, i, k): 1 \leq i \leq N, k \geq 1\},
$$

where some of the elements of this set are identified. In particular, $(A, i, 1)$ and $(B, i, 1)$ are identified and represent $[i] \in \mathscr{D}$. Furthermore $(A, i, k)$ and $(B, i, k)$ have the successors $(A, i, k+1)$ and $(B, i, k+1)$ respectively. All other successors of $(A, i, k)$ or $(B, i, k)$ are in $\{[i]: 1 \leq i \leq N\}$ except possibly one, which is $(B, j, l)$ or $(A, j, l)$ respectively, where $l \leq j \leq N$ and $l \leq k$. This shows that $\mathscr{D}_{n}=\{(A, i, k)$, $(B, i, k): 1 \leq i \leq N, 1 \leq k \leq n\}$.

If a closed path is contained in $\mathscr{A}_{i}=\{(A, i, k): k \geq 1\}$ for some $i$, then $(A, i, l)=$ $(A, i, m)$ for some $l \neq m$ and $(A, i, l+j)=(A, i, m+j)$ for $j \geq 0$ (cf. [2]). The path represents $\sigma^{l-1}\left(\underline{a}^{i}\right)=\sigma^{m-1}\left(\underline{a}^{i}\right) \in Z$ and no other closed path can be contained in $\mathscr{A}_{i}$. We collect the closed paths contained in some $\mathscr{A}_{i}$ or in some $\mathscr{B}_{i}=\{(B, i, k): k \geq 1\}$ as the exceptional set, which has then at most $2 N$ elements and suppose now that the path is not contained in an $\mathscr{A}_{i}$ or $\mathscr{B}_{i}$.

Let $(A, i, k)$ be that element of the closed path of length $n$, which has the largest $k$. Let $l \leq k$ be the smallest integer, such that $(A, i, l) \rightarrow(A, i, l+1) \rightarrow \cdots \rightarrow(A, i, k)$ is contained in the path. As $(A, i, l-1)$ is not the predecessor of $(A, i, l)$ in the path, there is a $(B, j, m)$ in the path with $(B, j, m) \rightarrow(A, i, l)$. It follows from lemma 12 of [2] that then $(B, j, t)$ has only the predecessor $(B, j, t-1)$ for $m-l+1<t \leq m$, so that

$$
(B, j, m-l+1) \rightarrow \cdots \rightarrow(B, j, m) \rightarrow(A, i, l) \rightarrow \cdots \rightarrow(A, i, k),
$$

which has length $k+1$, is contained in the closed path of length $n$. This implies $k \leq n-1$. As $(A, i, k)$ had the largest $k$ of all elements of the path and as $\mathscr{D}_{n}=$ $\{(A, i, k),(B, i, k): 1 \leq i \leq N, 1 \leq k \leq n\}$, we get (i).

Now (ii) follows from (i) and the definitions, where we choose $k_{0}$ such that the exceptional paths of (i) are in $\mathscr{D}_{k_{n}}$.

THEOREM 1. The convergence radius of $C(z)$ is at least $1 / r(M)$. For $|z|<1 / r(M)$ we have $\lim _{n \rightarrow \infty} C_{n}(z)=C(z)$ and $C(z)=\tilde{D}(z)^{-1}$.

Proof. We apply the formula det $\exp K=\exp \operatorname{tr} K$ to $K=\log \left(I-z M_{k}\right)$ and get

$$
C_{k}(z)=\exp \left(-\sum_{n \geq 1} \frac{z^{n}}{n} \operatorname{tr} M_{k}^{n}\right)
$$

for $|z|<1 / r(M)$ and $k \geq 1$ using the Taylor series of $\log (1-x)$. By (ii) of lemma 4 , the coefficients of $z^{n}$ on both sides of (2.1) converge for $k \rightarrow \infty$ to the coefficients of $z^{n}$ in $C(z)$ and $\tilde{D}(z)^{-1}$ respectively. Hence $C(z)$ has the same convergence radius as $\tilde{D}(z)^{-1}$ and the desired results follow.

Remarks. (i) Theorem 1 together with results of [8] shows that the kneading invariant, defined in [8] for continuous $T$, is the characteristic power series of the Markov diagram. 
(ii) One can show that theorem 1 also holds for topologically transitive subsets $\Omega$ of $\Sigma_{T}^{+}$.

We turn now to a different question. For a $\sigma$-invariant subset $X$ of $\Sigma_{T}^{+}$set $Z(X)=$ $\{n: \exists x \in X$ with period $n\}$. We want to find out what subsets of $\mathbb{N}$ can occur as $Z\left(\Sigma_{T}^{+}\right)$or $Z(\Omega)$, where $\Omega$ is a topologically transitive subset of $\Sigma_{T}^{+}$. One easily sees that every finite type subshift $(S, \sigma)$ can occur as $\left(\Sigma_{T}^{+}, \sigma\right)$ for some piecewise monotonic transformation $T$ : Introduce the lexicographic ordering in $S$. Then cylinder sets become closed intervals, on which $\sigma$ is increasing. Identifying endpoints of cylinder sets, $S$ becomes isomorphic to $[0,1]$ and $\sigma$ becomes a piecewise increasing $T$ on $[0,1]$, whose $\Sigma_{T}^{+}$is $S$. On the other hand, one can show that the set $Z(\Omega)$, if it is non-empty, always equals a set $Z(S)$, where $S$ is a finite type subshift contained in $\Omega$. Hence the sets $Z(\Omega)$, which occur for topologically transitive subsets $\Omega$ of some $\Sigma_{T}^{+}$, are exactly the sets $Z(S)$, which occur for finite type subshifts $S$.

In the next section, we consider this problem for a special class of piecewise increasing $T$.

\section{Monotonic rnod 1 transformations}

We call $T:[0,1) \rightarrow[0,1)$ monotonic $\bmod 1$ if $T(x)=h(x) \bmod 1$, where $h:[0,1) \rightarrow \mathbb{R}$ is continuous and increasing. Suppose $h(0) \in[0,1)$ and choose $N$ such that $\lim _{t \rightarrow 1} h(t) \in(N-1, N]$. We can split $[0,1)$ up into $N$ half open intervals $J_{i}$ with $T \mid J_{i}$ continuous and increasing. The shift space $\Sigma_{T}^{+}$is defined by (1.2). We consider 1 as a periodic point, if $\lim _{t \uparrow 1} T^{k}(t)=1$ for some $k$. Let $X \subset \Sigma_{T}^{+}$be $\sigma$-invariant and $L=\varphi^{-1}(X)$. Set $\underline{a}=\varphi(0)$ and $\underline{b}=\lim _{t \uparrow 1} \varphi(t)$. For monotonic mod 1 transformations one has $Y=\bigcup_{k=0}^{\infty} \sigma^{-k}\{\underline{a}, \underline{b}\}$, hence $Z(L)=Z(X)$ by lemma 1 , where $Z(X)$ and $Z(L)$ are the sets of $n$ such that points of period $n$ exist in $X$ or $L$ respectively.

In order to investigate $Z(X)$ we need more information about the Markov diagram of a monotonic mod 1 transformation, proved in [3], [4] and [6]. For $\underline{a}=a_{0} a_{1} a_{2} \cdots$ and $\underline{b}=b_{0} b_{1} b_{2} \cdots$ define integers $r_{1}, r_{2}, \ldots,\left(r_{i} \geq 1\right)$ and $s_{1}, s_{2}, \ldots,\left(s_{i} \geq 1\right)$ inductively such that

$$
a_{R_{k}+i}=b_{i-1} \quad \text { for } 1 \leq i \leq r_{k+1}-1, \quad a_{R_{k}+r_{k+1}} \neq b_{r_{k+1}-1},
$$

where $R_{k}=r_{1}+\cdots+r_{k}$ and $R_{0}=0$, and

$$
b_{s_{k}+i}=a_{i-1} \quad \text { for } 1 \leq i \leq s_{k+1}-1, \quad b_{S_{k}+s_{k+1}} \neq a_{s_{k+1}-1},
$$

where $S_{k}=s_{1}+\cdots+s_{k}$ and $S_{0}=0$. Set $E_{i}=[i], \quad A_{1}=[1], \quad B_{1}=[N]$ and $A_{j+1}=\sigma\left(A_{j}\right) \cap\left[a_{j}\right], B_{j+1}=\sigma\left(B_{j}\right) \cap\left[b_{j}\right]$ for $j \geq 1$. It is shown in [3] and [6], that $\mathscr{D}=\left\{E_{i}, A_{j}, B_{j}: 2 \leq i \leq N-1, j \geq 1\right\}$ and that there are the following arrows in the Markov diagram.

$$
\begin{array}{cccc} 
& A_{m} \rightarrow A_{m+1}, & B_{m} \rightarrow B_{m+1} \quad \text { for } m \geq 1, \\
A_{R_{k}} \rightarrow B_{r_{k}}, & A_{R_{k}} \rightarrow E_{l} & \left(a_{R_{k}}<l<b_{r_{k}-1}\right) & \text { for } k \geq 1, \\
B_{S_{k}} \rightarrow A_{s_{k}}, \quad B_{S_{k}} \rightarrow E_{l} & \left(a_{s_{k}-1}<l<b_{S_{k}}\right) & \text { for } k \geq 1, \\
E_{k} \rightarrow E_{l} \quad(2 \leq l \leq N-1), & E_{k} \rightarrow A_{1}, B_{1} \quad \text { for } 2 \leq k \leq N-1 .
\end{array}
$$

We have $A_{m} \subset\left[a_{m-1}\right], B_{m} \subset\left[b_{m-1}\right]$ and $E_{m}=[m]$, hence $\psi\left(A_{m}\right)=a_{m-1}, \psi\left(B_{m}\right)=b_{m-1}$ and $\psi\left(E_{m}\right)=m$ (cf. $\S 1$ ). If $A_{m}=A_{n}$ for $m<n$, then $A_{m+k}=A_{n+k}$ for $k \geq 0$, since 
$\boldsymbol{A}_{l+1}$ is uniquely determined as that successor of $\boldsymbol{A}_{l}$ to which $\psi$ assigns the minimal number. As $\psi\left(A_{l}\right)=a_{I-1}$, we get $\sigma^{m-1}(\underline{a})=\sigma^{n-1}(\underline{a})$. If $r_{l}<\infty$ for all $l$, then there is a $k \geq 0$ such that $A_{m+k}=A_{n+k}$ has more than one successor and hence $m+k=R_{i}$ and $n+k=R_{i+j}$ for some $i, j>0$ by (3.3) and (3.4). We have

$$
A_{R_{i}+1}=A_{R_{i+j}+1} \text { and } \sigma^{R_{i}}(\underline{a})=\sigma^{R_{i+j}}(\underline{a}) \text {. }
$$

A similar statement holds if $B_{m}=B_{n}$ for some $m \neq n$. If $r_{i+1}=\infty$ and $s_{j+1}=\infty$, it follows from (3.1) and (3.2) that $\sigma^{R_{i}+1} \underline{a}=\underline{b}$ and $\sigma^{s_{j}+1} \underline{b}=\underline{a}$ and from this that (cf. (1.7) of [6])

$$
A_{R_{i}+S_{j}+2}=B_{S_{j}+1}, \quad B_{S_{j}+R_{i}+2}=A_{R_{i}+1} .
$$

After this preparation we now begin the investigation of $Z(\Omega)$ for some topologically transitive subset $\Omega$ of $\Sigma_{T}^{+}$. For a subset $\mathscr{F}$ of $\mathscr{D}$ set

$$
Z(\mathscr{F})=\{n \text { : there is a closed path of length } n \text { in } \mathscr{F}\} \text {. }
$$

A maximal irreducible subset $\mathscr{D}^{\prime}$ of $\mathscr{D}$ determines $\Omega$ (cf. [5]). We consider first $Z\left(\mathscr{D}^{\prime}\right)$ (cf. lemma 2). By (3.5) we have either

$$
\left\{E_{k}: 2 \leq k \leq N-1\right\} \subset \mathscr{D}^{\prime}
$$

or

$$
\left\{E_{k}: 2 \leq k \leq N-1\right\} \cap \mathscr{D}^{\prime}=\varnothing .
$$

We consider first the easier case (3.8). If $N \geq 4$, then $Z\left(\left\{E_{k}: 2 \leq k \leq N-1\right\}\right)=\mathbb{N}$, hence $Z\left(\mathscr{D}^{\prime}\right)=Z(\mathscr{D})=\mathbb{N}$. If $N=3$ and $\mathscr{D}^{\prime}=\left\{E_{2}\right\}$, then $Z\left(\mathscr{D}^{\prime}\right)=\{1\}$. If $\mathscr{D}^{\prime}$ is larger than $\left\{E_{2}\right\}$, let $m$ be the smallest integer with $A_{m} \rightarrow E_{2}$ or $B_{m} \rightarrow E_{2}$. Suppose $A_{m} \rightarrow E_{2}$. By lemma 9 of [4] we have $\underline{a}=122 \cdots 21 \cdots$ and the Markov diagram looks like this $\left(s_{1}=3\right)$ :

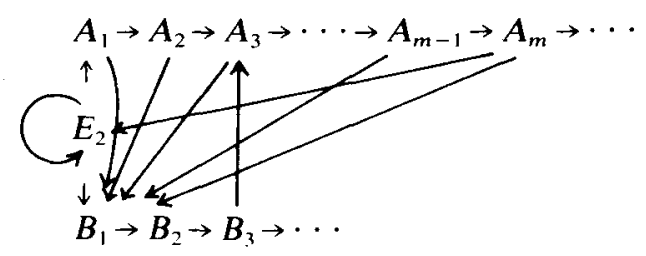

We need the following. Set $\mathcal{N}=\mathbb{N}^{2} \cup\{(1,0),(0,1)\}$. Suppose $\mathscr{D}^{\prime}$ contains two closed paths of length $k$ and $l$ with non-empty intersection. Then

$$
v k+w l \in Z\left(\mathscr{D}^{\prime}\right) \quad \text { for }(v, w) \in \mathcal{N} \text {. }
$$

One gets a closed path of length $v k+w l$, if one runs $v$ times through the path of length $k$ and then $w$ times through the path of length $l$. In the above diagram the paths $E_{2} \rightarrow A_{1} \rightarrow \cdots \rightarrow A_{m} \rightarrow E_{2}$ and $E_{2} \rightarrow E_{2}$ are not disjoint, hence $\{1, m+1, m+2$, $\cdots\} \subset Z\left(\mathscr{D}^{\prime}\right)$ by (3.10). If $s_{1} \leq m$, the closed path $B_{1} \rightarrow \cdots \rightarrow B_{s_{1}} \rightarrow A_{s_{1}} \rightarrow A_{s_{1}+1} \rightarrow \cdots \rightarrow$ $A_{i} \rightarrow B_{1}$ has length $i+1$ for $s_{1} \leq i \leq m-1$. Hence we get (for $n \notin Z(\mathscr{D})$, if $2 \leq n<$ $\min \left\{m, s_{1}\right\}$, we use (i) of lemma $4,(3.6)$ and lemma 5 below)

$$
Z\left(\mathscr{D}^{\prime}\right)=Z(\mathscr{D})=\{1\} \cup\left\{n: n>\min \left\{m, s_{1}\right\}\right\} .
$$

We can write this as $\{v p+w q:(v, w) \in \mathcal{N}\}$ with $p=1$ and $q=\min \left\{m, s_{1}\right\}+1$. Then it fits into the notation of theorem 2 below. In the case $B_{m} \rightarrow E_{2}$ we get the same 
result with $p=\min \left\{m, r_{1}\right\}+1$ and $q=1$. This completes the case (3.8), since there are no $E_{k}$ in the case $N=2$.

It remains to investigate (3.9). To this end we need two more lemmas about the numbers $r_{i}$ and $s_{i}$ proved in [3] and [4].

Lemma 5. Setting $R_{0}=S_{0}=0$ and $R_{\infty}=S_{\infty}=\infty$, there is for every $m \geq 1$ a $P(m)$ and a $Q(m)$ with $0 \leq P(m), Q(m) \leq \infty$ such that $r_{m}=1+S_{P(m)}$ and $s_{m}=1+R_{Q(m)}$.

By (3.3) for a $\mathscr{D}^{\prime}$ satisfying (3.9) there exist $q, p, q^{\prime}, p^{\prime}$ with $1 \leq q<q^{\prime} \leq \infty$ and $1 \leq p<p^{\prime} \leq \infty$ such that $\mathscr{D}^{\prime}=\left\{A_{i}, B_{j}: q \leq i<q^{\prime}, p \leq j<p^{\prime}\right\}$. Set $\overline{\mathscr{D}}=\left\{A_{i}, B_{j}: q \leq i\right.$, $p \leq j\}$. The set $F \subset \Sigma_{T}^{+}$of all one-sided paths in $\overline{\mathscr{D}}$ is a $\sigma$-invariant finite union of intervals containing $\Omega$ (cf. [5], [6]).

Lemma 6. Suppose $\mathscr{D}^{\prime}$ satisfies (3.9). Let $m$ be such that $A_{R_{m}} \in \overline{\mathscr{D}}\left(B_{S_{m}} \in \overline{\mathscr{D}}\right)$ and that $r_{m}>p\left(s_{m}>q\right)$. Then there is a $j, 1 \leq j \leq \infty$ with $r_{m+i}=r_{Q(P(m))+i}\left(s_{m+i}=s_{P(Q(m))+i}\right)$ for $1 \leq i<j$ and $r_{m+j}>r_{Q(P(m))+j}\left(s_{m+j}>s_{Q(P(m))+j}\right)$.

We use lemma 6 to find a closed path in $\mathscr{D}^{\prime}$. By the irreducibility of $\mathscr{D}^{\prime}$, there must be a $D \in \mathscr{D}^{\prime}$ with $D \rightarrow A_{q}$. Hence by (3.4), $q=s_{h}$ for some $h$. Similarly $p=r_{g}$ for some $g$. We choose $g$ and $h$ minimal. By lemma 5 , we have $q=s_{h}=1+R_{i}, i=Q(h)$. We consider $r_{i+1}$. As $\overline{\mathscr{D}}=\left\{A_{l}, B_{j}: l \geq q, j \geq p\right\}$, and $\mathscr{D}^{\prime}$ is irreducible, $r_{i+1}>p$ implies $1+S_{P(i+1)}>p$ and $Q(P(i+1)) \geq i$, hence $r_{i+2}>p$ by lemma 6 . The same argument gives that $r_{i+2}>p$ implies $r_{i+3}>p$ and so on, a contradiction to $r_{g}=p$ and $g>i$, which holds as $A_{R_{g}} \in \overline{\mathscr{D}}$. Hence $r_{i+1}=p$ and $g=i+1$, i.e. $s_{h}=1+R_{g-1}=q$. Similarly one gets $r_{g}=1+S_{h-1}=p$ (cf. [6]). We have the following closed path (cf. (3.3) and (3.4)).

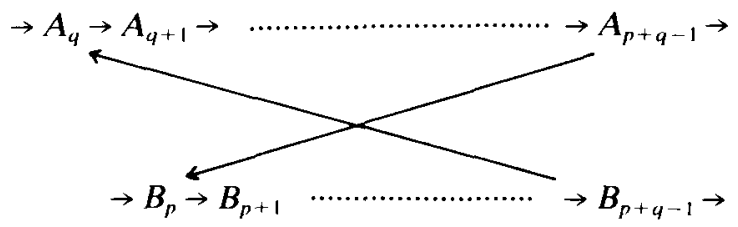

We say that a pair $(i, j)$ gives rise to a closed path in $\mathscr{D}$, if $r_{i}<1+S_{j}$ and $s_{j}<1+R_{i}$. The closed path is given by $A_{s_{j}} \rightarrow \cdots \rightarrow A_{R_{i}} \rightarrow B_{r_{i}} \rightarrow \cdots \rightarrow B_{S_{j}} \rightarrow A_{s_{j}}$ and has length $R_{i-1}+S_{j-1}+2$. Hence the pair $(g, h)$ gives rise to the above closed path of length $p+q$. If

$$
\mathscr{D}^{\prime}=\left\{A_{i}, B_{j}: q \leq i<p+q, p \leq j<p+q\right\}
$$

then $Z\left(\mathscr{D}^{\prime}\right)=\{p+q\}$.

If (3.12) is not satisfied, then we have

$$
\mathscr{D}^{\prime} \supsetneq\left\{A_{i}, B_{j}: q \leq i<p+q, p \leq j<p+q\right\} .
$$

By (3.4) there must then be a $k>g$ with $p \leq r_{k}<p+q$, or a $k>h$ with $q \leq s_{k}<p+q$, since $\mathscr{D}^{\prime}$ is irreducible. By lemma 5 , we have then $r_{k}=p$ or $s_{k}=q$ respectively. Let $t$ be such that $r_{i} \leq p+q$ for $g \leq i<t$ and $r_{t}>p+q$. If $r_{i} \leq p+q$ for all $i \geq g$, we set $t=\infty$. Let $u$ be such that $s_{i} \leq p+q$ for $h \leq i<u$ and $s_{u}>p+q$. If $r_{i}=p+q$ for $g<i<t$ and $s_{i}=p+q$ for $h<i<u$, it follows from lemma 6 by induction ( $m$ such 
that $r_{m}>p+q$ or $\left.s_{m}>p+q\right)$ that $r_{i} \geq p+q$ for all $i>g$ and $s_{i} \geq p+q$ for all $i>h$, which implies (3.12). As (3.12) does not hold, at least one of the following two statements occurs:

$$
\begin{aligned}
& r_{i}=p+q \text { for } g<i<\tilde{g} \text { and } \quad r_{\tilde{g}}=p \text { for some } \tilde{g} \text { with } g<\tilde{g}<t \\
& s_{i}=q+p \text { for } h<i<\tilde{h} \text { and } s_{\tilde{h}}=q \text { for some } \tilde{h} \text { with } h<\tilde{h}<u .
\end{aligned}
$$

In order to investigate $Z\left(\mathscr{D}^{\prime}\right)$, we define a vector $\underline{d}_{i}$ for every $i$ with $r_{g+i-1} \leq p+q$. For $i \geq 1$ set $\underline{d}_{i}=(1,0)$, if $r_{g+i-1}=p$, and $\underline{d}_{i}=(1,1)$, if $r_{g+i-1}=p+q$. Furthermore set $\underline{c}_{0}=(0,1)$ and for $1 \leq i \leq t-g$ set $\underline{c}_{i}=(0,1)+\underline{d}_{1}+\cdots+\underline{d}_{i}$. As $g+i-1<t$, these $\underline{c}_{i}$ are defined. We have $1+R_{g+i-1}=v p+w q$ for $0 \leq i \leq t-g$, where $(v, w)=\underline{c}_{i}$. We need this for the following reason: If $r_{g+i-1}=p$, the pair $(g+i-1, h)$ gives rise to a closed path $\mathscr{R}_{i}$ in $\mathscr{D}^{\prime}$ of length $1+R_{g+i-1}=v p+w q$.

The slope of a vector $(v, w)$ is defined as $w / v$. We write $\underline{c} \leq \tilde{c}$ if the slope of $c$ is less than or equal to the slope of $\underline{\tilde{c}}$, and $\underline{c}<\underline{\tilde{c}}$ if the slope of $\tilde{\boldsymbol{c}}$ is less than the slope of $\underline{c}$. Set $\lambda=\inf \left\{\right.$ slope of $\left.\underline{c}_{i}: 1 \leq i \leq t-g\right\}$ and $H=\left\{(v, w) \in \mathbb{N}^{2}: \lambda<w / v<1\right\}$. Remark that $\lambda<1$ if and only if (3.13) holds, otherwise $\lambda=1$. We want to show $\{v p+w q:(v, w) \in H\} \subset Z\left(\mathscr{D}^{\prime}\right)$. To this end we need some lemmas.

LEMMA 7. Choose $i$ such that $\underline{d}_{i}$ is defined and let $m$ be the largest element of $\mathbb{N} \cup\{\infty\}$, such that $\underline{d}_{j}$ and $\underline{d}_{i+j}$ are defined for $1 \leq j<m$. Then either $\underline{d}_{i+j}=\underline{d}_{j}$ for $1 \leq j<m$ or there is a $k<m$ with $\underline{d}_{i+j}=\underline{d}_{j}$ for $1 \leq j<k$ and $\underline{d}_{i+k}>\underline{d}_{k}$, i.e. $\underline{d}_{i+k}=(1,1)$ and $\underline{d}_{k}=$ $(1,0)$.

Proof. As $\underline{d}_{1}=(1,0)$, we have $k=1$ if $\underline{d}_{i+1}=(1,1)$. Hence suppose $\underline{d}_{i+1}=(1,0)$. If $\underline{d}_{i}=(1,0)$ the result follows, because the largest integer $l$ with $r_{g+j-1}=p$ for $1 \leq j \leq l$ is greater than or equal to the largest integer $n$ with $r_{g+i+j-2}=p$ for $1 \leq j \leq n$, by lemma 6. If $\underline{d}_{i}=(1,1)$ then $r_{g+i-1}=p+q$ and $Q(P(g+i-1))=g-1$, so that the result follows again from lemma 6.

LEMMA 8. If $\underline{c}_{i}$ satisfies $\underline{c}_{i}=\underline{c}_{i-1}+(1,1)$, i.e. $\underline{d}_{i}=(1,1)$, and $\underline{c}_{i-1}+(1,0) \geq \underline{c}_{j}$, where $j<i$ is such that $\underline{c}_{n} \geq \underline{c}_{j}$ for $n \leq i-1$, then $\underline{c}_{n} \geq \underline{c}_{j}$ for all $n \leq t-g$.

Proof. By lemma 7, there exists a $k$ with $\underline{d}_{i+n}=\underline{d}_{n}$ for $1 \leq n \leq k$ and, if $i+k<t-g$, $\underline{d}_{i+k}>\underline{d}_{k}$, i.e. $\underline{d}_{i+k}=(1,1)$ and $\underline{d}_{k}=(1,0)$. Hence $\underline{c}_{n}=\underline{c}_{i-1}+(1,1)+\underline{d}_{1}+\cdots+\underline{d}_{n-i}$ for $i \leq n<i+k$, hence $\underline{c}_{n}=\underline{c}_{i-1}+(1,0)+\underline{c}_{n-i}$. Suppose $\underline{c}_{n}<\underline{c}_{j}$. As $\underline{c}_{i-1}+(1,0) \geq \underline{c}_{j}$, this implies $\underline{c}_{n-i}<\underline{c}_{j}$. If $n-i \geq i$, we get by the same argument that $\underline{c}_{n-2 i}<\underline{c}_{j}$ and so on. Let $l$ be such that $0 \leq n-l i \leq i-1$ and we get $\underline{c}_{n-l i}<\underline{c}_{j}$, a contradiction to our assumptions. Hence $\underline{c}_{n} \geq \underline{c}_{j}$ for $i \leq n<i+k$. If $i+k=t-g$, the lemma is proved. Otherwise $\underline{c}_{i+k-1}+(1,0)=\underline{c}_{i-1}+(1,1)+\underline{d}_{1}+\cdots+\underline{d}_{k} \geq \underline{c}_{j}$ follows as above for $\underline{c}_{n}$, since $\underline{d}_{k}=(1,0)$. As $\underline{d}_{i+k}=(1,1)$, the assumptions of the lemma are satisfied for $i+k$ instead of $i$. Hence induction finishes the proof.

LEMMA 9. (i) Let $i<j$. If $\underline{c}_{i}>\underline{c}_{j}$, then $\underline{c}_{j}-\underline{c}_{i}<\underline{c}_{j}$. If $\underline{c}_{i} \leq \underline{c}_{j}$ then $\underline{c}_{j}-\underline{c}_{i} \geq \underline{c}_{i}$.

(ii) Let $m<n$. Suppose that $\underline{c}_{m}>\underline{c}_{n}$ and $\underline{c}_{i} \geq \underline{c}_{m}$ for $i \leq n-1$. If there is a $k$ with $1 \leq k \leq n-m-1$ such that $\underline{d}_{n+j}=\underline{d}_{m+j}$ for $1 \leq j<k$ and $\underline{d}_{n+k}>\underline{d}_{m+k}$, then $\underline{c}_{j} \geq \underline{c}_{n}$ for all $i \leq t-g$.

(iii) Let $n$ be such that $\underline{c}_{i} \geq \underline{c}_{n}$ for all $i<n$. If there is a $k$ with $1 \leq k \leq n-1$, such that $\underline{d}_{n+1}=(1,1), \underline{d}_{n+j}=\underline{d}_{j}$ for $1<j<k$, and $\underline{d}_{n+k}>\underline{d}_{k}$, then $\underline{c}_{i} \geq \underline{c}_{n}$ for all $i \leq t-g$. 
Proof. As $\underline{c}_{i}, \underline{c}_{j}$ and $\underline{c}_{j}-\underline{c}_{i}$ are vectors with non-negative entries, (i) follows from the definition of the order relation.

We show (ii). For $j<k$ we have

$$
\begin{aligned}
\underline{c}_{n+j} & =\underline{c}_{n}+\underline{d}_{n+1}+\cdots+\underline{d}_{n+j}=\underline{c}_{n}+\underline{d}_{m+1}+\cdots+\underline{d}_{m+j} \\
& =\underline{c}_{n}+\underline{c}_{m+j}-\underline{c}_{m}>\underline{c}_{n},
\end{aligned}
$$

because $\underline{c}_{m+j}-\underline{c}_{m} \geq \underline{c}_{m}$ by (i). The same computation for $j=k-1$ gives $\underline{c}_{n+k-1}+$ $(1,0)=\underline{c}_{n}+\underline{c}_{m+k}-\underline{c}_{m}$ since $\underline{d}_{m+k}=(1,0)$. Using $\underline{c}_{m+k} \geq \underline{c}_{m}$ and (i), we get $\underline{c}_{n+k-1}+$ $(1,0)>\underline{c}_{n}$. Now (ii) follows from lemma 8 for $j=n$ and $i=n+k$, as $\underline{d}_{n+k}=(1,1)$.

The proof of (iii) is the same as that of (ii) setting $m=0$. One gets $\underline{c}_{n+j}=\underline{c}_{n}+\underline{c}_{j} \geq \underline{c}_{n}$ for $1 \leq j<k$ and $\underline{c}_{n+k-1}+(1,0)=\underline{c}_{n}+\underline{c}_{k}>\underline{c}_{n}$.

We call a vector $\underline{c}_{i}$ minimal if $\underline{c}_{j}>\underline{c}_{i}$ for $0 \leq j<i$. Let $\underline{e}_{0}=\underline{c}_{0}>\underline{e}_{1}=\underline{c}_{1}>\underline{e}_{2}>\underline{e}_{3}>\cdots$ be the finite or countable subsequence of $\left(\underline{c}_{i}\right)$ consisting of minimal vectors. Then we have:

Proposition 1. Let $i \geq 1$ be such that $\underline{e}_{i+1}$ exists. Then there is an integer $m_{i} \geq 2$ with $\underline{e}_{i+1}=m_{i} e_{i}-\underline{e}_{i-1}$. Furthermore for $1 \leq k \leq m_{i}-1$ there is a $j$ with $k \underline{e}_{i}=\underline{c}_{j}$, where $\underline{d}_{j}=(1,0)$, if $i \geq 2$.

Proof. Let $v$ and $w$ be such that $\underline{e}_{i-1}=\underline{c}_{v}$ and $\underline{e}_{i}=\underline{c}_{w}$. We show by induction that for $i \geq 2$ :

(i) $\underline{e}_{i}=m \underline{e}_{i-1}-\underline{e}_{i-2}$ for some $m \geq 2$ and $\underline{d}_{w}=(1,0)$;

(ii) $i>2,2 \leq k \leq m-1 \Rightarrow k \underline{e}_{i-1}=\underline{c}_{j}$ for some $j$ with $\underline{d}_{j}=(1,0)$;

(iii) $\underline{d}_{w-v}=(1,1)$, if $w-v>1$, and $\underline{d}_{w-v+j}=\underline{d}_{j}$ for $1 \leq j \leq v$;

(iv) $\underline{d}_{v+1}=(1,1)$, if $w-v>1$, and $\underline{d}_{v+j}=\underline{d}_{j}$ for $1<j<w-v$.

We check first (i)-(iv) for $i=2$. Let $m$ be such that $\underline{d}_{i}=(1,1)$ for $1<i<m$ and $\underline{d}_{m}=(1,0)$. Then $\underline{c}_{i} \geq \underline{c}_{1}$ for $i<m$ and $\underline{c}_{m}<\underline{c}_{1}$. Hence $\underline{e}_{2}=\underline{c}_{m}=m \underline{e}_{1}-\underline{e}_{0}$. If no such $m$ exists, we have also no $\underline{e}_{2}$. As $v=1$ and $w=m$, (iii) and (iv) follow from $\underline{d}_{i}=(1,1)$ for $1<i<m$ and $\underline{d}_{m}=(1,0)$.

Now suppose that (i)-(iv) hold for $i$. We prove them for $i+1$ supposing that $\underline{e}_{i+1}$ exists. It follows from (iii) and lemma 7, that there is a $k \geq 1$ with $\underline{d}_{v+j}=\underline{d}_{w+j}$ for $1 \leq j<k$ and, if $w+k<t, \underline{d}_{v+k}<\underline{d}_{w+k}$. As $\underline{c}_{v}=\underline{e}_{i-1}, \underline{c}_{w}=\underline{e}_{i}$, and $\underline{e}_{i+1}$ exists, it follows from (ii) of lemma 9 with $m=v$, and $n=w$ that $k \geq w-v$, i.e.

$$
\underline{d}_{w+j}=\underline{d}_{v+j} \quad \text { for } 1 \leq j<w-v .
$$

Hence $\underline{c}_{w+j}=\underline{c}_{w}+\underline{c}_{v+j}-\underline{c}_{v}>\underline{c}_{w}$, by (i) of lemma 9 . If we also have

$$
\underline{d}_{2 w-v}=\underline{d}_{w}, \quad \text { which is }(1,0) \text { by (i), }
$$

then $\underline{c}_{2 w-v}=\underline{c}_{w}+\underline{c}_{w}-\underline{c}_{v}<\underline{c}_{w}$ by (i) of lemma 9. Hence $\underline{e}_{i+1}=\underline{c}_{2 w-v}=2 \underline{e}_{i}-\underline{e}_{i-1}$. Together with $\underline{d}_{2 w-v}=(1,0)$ this gives (i) for $i+1$. As $m=2$, (ii) is an empty statement. Let $v^{\prime}, w^{\prime}$ be such that $\underline{e}_{i}=\underline{c}_{v^{\prime}}$ and $\underline{e}_{i+1}=\underline{c}_{w^{\prime}}$, i.e. $v^{\prime}=w$ and $w^{\prime}=2 w-v$. As $w^{\prime}-v^{\prime}=$ $w-v$, (iii) and (iv) for $i+1$ follow from (iii) and (iv) for $i,(3.15)$ and (3.16).

Now we consider the case where (3.16) does not hold. Suppose that for some $l \geq 1$

$$
\begin{gathered}
\underline{e}_{i}=\underline{c}_{l w}, \quad \underline{c}_{i} \geq \underline{c}_{w} \text { for } i \leq l w, \\
\underline{d}_{l w+j}=\underline{d}_{v+j} \text { for } 1 \leq j<w-v, \quad \underline{d}_{l w+w-v}=(1,1)
\end{gathered}
$$


For $l=1$ this is (3.15) and the converse of (3.16). It follows from lemma 7 that there is a $k \geq 1$ with

$$
\underline{d}_{l w+w-v+j}=\underline{d}_{j} \text { for } 1 \leq j<k, \quad \underline{d}_{l w+w-v+k}>\underline{d}_{k}
$$

Now we get

$$
\underline{d}_{l w+1}=(1,1), \quad \underline{d}_{l w+j}=\underline{d}_{j} \text { for } 1<j<\min \{2 w-v, w-v+k\} .
$$

For $j \leq w-v$ this follows from (3.17) and (iv), for $w-v<j \leq w$ from (3.18) and (iii) and for $j>w$ from (3.18) and (3.15). It follows from (iii) of lemma 9 with $n=l w,(3.18)$ and the existence of $\underline{e}_{i+1}$, that $k \geq w$. Hence we get from (3.19) that $\underline{c}_{l w+j}=\underline{c}_{l w}+\underline{c}_{j} \geq \underline{c}_{w}$ for $j<w$ and $\underline{c}_{l w+w}=\underline{c}_{l w}+\underline{c}_{w}=(l+1) \underline{e}_{i \cdot}$. Furthermore $\underline{d}_{l w+w}=\underline{d}_{w}=$ $(1,0)$, which shows (ii). As $k \geq w$, we have by (3.19) that $\underline{d}_{l w+j}=\underline{d}_{j}$ for $w<j<2 w-v$. If $\underline{d}_{l w+2 w-v}=(1,1)$, all requirements of (3.17) are satisfied for $l+1$ instead of $l$ and we can perform the whole argument again. Otherwise $\underline{d}_{l w-2 w-v}=(1,0)$ and $\underline{c}_{l w+2 w-v}=$ $(l+1) \underline{e}_{i}+\underline{e}_{i}-\underline{e}_{i-1}$ (cf (3.19) and (3.15)). By (i) of lemma 9, we get $\underline{e}_{i+1}=\underline{c}_{l w+2 w-v}$. Hence (i) is shown with $m=l+2$. If $v^{\prime}$ and $w^{\prime}$ are such that $\underline{c}_{v^{\prime}}=\underline{e}_{i}$ and $\underline{c}_{w^{\prime}}=\underline{e}_{i+1}$, then $v^{\prime}=w, w^{\prime}=m w-v$, and

$$
w^{\prime}-v^{\prime}=(m-1) w-v
$$

As $k \geq w$, we get (iii) for $i+1$ from (3.18) and (3.20). If $w-v=1$, then $\underline{d}_{w^{\prime}-v^{\prime}}=\underline{d}_{l w+1}=$ $(1,1)$ by (3.19). We get (iv) for $i+1$ from (3.19) for $l=1,2, \ldots, m-2$. This finishes the induction step.

As we have defined $\underline{d}_{i}$ for $r_{\mathrm{g}+i-1}$, we define $\underline{d}_{i}^{\prime}=(0,1)$ if $s_{h+i-1}=q, \underline{d}_{i}^{\prime}=(1,1)$ if $s_{h+i-1}=q+p$, and $\underline{c}_{i}^{\prime}=(1,0)+\underline{d}_{1}^{\prime}+\cdots+\underline{d}_{i}^{\prime}$ for $0 \leq i \leq u-h$. Again we have $1+S_{i+h-1}=v p+w q$ with $(v, w)=\underline{c}_{i}^{\prime}$. Using lemma 5 and induction, we also get representations of $1+R_{i+g-1}$ for $i>t-g$ and of $1+S_{i+h-1}$ for $i>u-h$ as $v p+w q$. We use these representations in the sequel. Set $\mu=\max$ \{slope of $\underline{c}_{i}^{\prime}: 1 \leq i \leq u-h$ \} and $H^{\prime}=\{(v, w): 1<w / v<\mu\}$. We set $K=\{(v, w): \lambda<w / v<\mu\}$. As either (3.13) or (3.14) occurs, we have either $\lambda<1$ or $\mu>1$, so that $K \neq \varnothing$. We shall show that

$$
\{v p+w q:(v, w) \in K\} \subset Z\left(\mathscr{D}^{\prime}\right) .
$$

We consider first $K_{\alpha}=\left\{(v, w) \in \mathbb{N}^{2}: w / v=\alpha\right\}$ for $\alpha=1$.

LEMMA 10. If $\mu>1$, i.e. (3.14) holds, and $r_{g+1}=1+S_{h+j}$ with $-1 \leq j<\tilde{h}-h$, then $k(p+q) \in Z\left(\mathscr{D}^{\prime}\right)$ for $k \geq z:=j+2$.

Proof. As $r_{g+1}<1+S_{\tilde{h}}$ and $s_{\tilde{h}}=q$, the pair $(g+1, \tilde{h})$ gives rise to a closed path in $\mathscr{D}^{\prime}$ of length $1+R_{\mathrm{g}}+1+S_{\tilde{h}-1}=p+q+(\tilde{h}-h)(p+q)$ (cf. (3.14)), which is not disjoint from the closed path of length $p+q$ given by $(g, h)$. By $(3.10)$, we have $k(p+q) \in$ $Z\left(\mathscr{D}^{\prime}\right)$ for $k \geq \tilde{h}-h+1$.

As $s_{h+i}=p+q$ for $1 \leq i<\tilde{h}-h$ and $r_{g+1}=1+S_{h+j}$, the pair $(g+1, h+i)$ gives rise to a closed path of length $1+R_{\mathrm{g}}+1+S_{h+i-1}=p+q+i(p+q)$ if $i>j$, hence $k(p+q) \in Z\left(\mathscr{D}^{\prime}\right)$ for $z \leq k \leq \tilde{h}-h$.

Proposition 2. $\{v p+w q:(v, w) \in K\} \subset Z\left(\mathscr{D}^{\prime}\right)$

Proof. We consider first $(v, w) \in H$. Suppose that $(v, w)$ is in an angle spanned by $\underline{e}_{i}$ and $\underline{e}_{i+1}$ for $i \geq 1$. As $\underline{e}_{0}=(1,0)$ and $\underline{e}_{1}=(1,1)$, it follows by induction from proposition 1 that $\operatorname{det}\left(\underline{e}_{i}, \underline{e}_{i+1}\right)=1$. Hence $(v, w)=j \underline{e}_{i}+k \underline{e}_{i+1}$ with $j, k \in \mathbb{N}$. Suppose $\underline{e}_{i}=\underline{c}_{m}$ 
and $\underline{e}_{i+1}=\underline{c}_{n}$. By proposition 1 , we have $\underline{d}_{m}=\underline{d}_{n}=(1,0)$. Hence the closed paths $\mathscr{R}_{m}$ and $\mathscr{R}_{n}$ of lengths $v^{\prime} p+w^{\prime} q$ with $\left(v^{\prime}, w^{\prime}\right)=\underline{c}_{m}$ and $v^{\prime} p+w^{\prime} q$ with $\left(v^{\prime}, w^{\prime}\right)=\underline{c}_{n}$ are not disjoint, so that we get a closed path of length $v p+w q$ by $(3.10)$, i.e. $v p+w q \in$ $Z\left(\mathscr{D}^{\prime}\right)$.

Now suppose $(v, w)=j \underline{e}_{i}$ for some $i>1$, such that $\underline{e}_{i+1}$ exists. It follows from proposition 1 that $j \underline{e}_{i}=\underline{c}_{n}$ for $1 \leq j<m_{i}$ with $\underline{d}_{n}=(1,0)$. Hence $\mathscr{R}_{n}$ is a closed path of length $v p+w q$. Suppose $\underline{e}_{i-1}=\underline{c}_{k}$ and $\underline{e}_{i+1}=\underline{c}_{l}$. As $\underline{d}_{k}=\underline{d}_{l}=(1,0)$, the paths $\mathscr{R}_{k}$ and $\mathscr{R}_{l}$ are not disjoint. As $\underline{e}_{i-1}+\underline{e}_{i+1}=m_{i} \underline{e}_{i}$ by proposition 1 , we get a closed path of length $v p+w q$ for $j=m_{i}$, by (3.10). This closed path is not disjoint from $\mathscr{R}_{n}$, where $\underline{c}_{n}=\underline{e}_{i}$, so that we get again by (3.10) a closed path of length $v p+w q$ for all $j>m_{\text {. }}$

If $(v, w) \in H^{\prime}$ the same proof works. If $\lambda<1<\mu$, we have $K_{1} \subset K$. We have to show $k(p+q) \in Z\left(\mathscr{D}^{\prime}\right)$ for $k \geq 1$. For $k=1$ we have the closed path given by the pair $(g, h)$, which has length $p+q$. For $k>1, k(p+q) \in Z\left(\mathscr{D}^{\prime}\right)$ follows from lemma 10 , because $\lambda<1$ implies (3.13), which gives $j=-1$ or 0 in lemma 10 .

We want to prove an inclusion in the other direction as in proposition 2. To this end we need some lemmas about $1+R_{i}$ for $i \geq g$ and $1+S_{i}$ for $i \geq h$.

LEMMA 11. Suppose that $r_{i}<\infty$ and $s_{i}<\infty$ for all $i$.

(i) $1+R_{i}=v p+w q$ with $(v, w) \in K_{1}$ for $g \leq i<\tilde{g},(v, w) \in K \cup K_{\lambda}$ for $\tilde{g} \leq i<t$ and $(v, w) \in K$ for $i \geq t$, if $\mu>1$, i.e. (3.14) holds, and with $(v, w) \in K \cup K_{1}$ for $i \geq h$, if $\lambda=1$. If $i$ is such that $r_{i}>p+q$ we have $R_{i+j}-R_{i-1}=v p+w q$ with $(v, w) \in K \cup K_{1}$ for $j \geq 1$ and $(v, w) \in K \cup K_{1} \cup K_{\mu}$ for $j=0$, where $\mu=1$ and $(v, w) \in K_{1}$ for some $j \geq 1$ imply $(v, w) \in K_{1}$ for $j=0$.

(ii) $1+S_{i}=v p+w q$ with $(v, w) \in K_{1}$ for $h \leq i<\tilde{h},(v, w) \in K \cup K_{\mu}$ for $\tilde{h} \leq i<u$, and $(v, w) \in K$ for $i \geq u$, if $\mu>1$, i.e. (3.14) holds, and with $(v, w) \in K \cup K_{1}$ for $i \geq h$, if $\mu=1$. If $i$ is such that $s_{i}>p+q$ we have $S_{i+j}-S_{i-1}=v p+w q$ with $(v, w) \in K \cup K_{1}$ for $j \geq 1$ and $(v, w) \in K \cup K_{1} \cup K_{\lambda}$ for $j=0$, where $\lambda=1$ and $(v, w) \in K_{1}$ for some $j \geq 1$ imply $(v, w) \in K_{1}$ for $j=0$.

Proof. We use induction. Let $t_{1}=t<t_{2}<\cdots$ and $u_{1}=u<u_{2}<\cdots$ be such that $r_{t_{i}}>p+q, s_{u_{i}}>p+q$ for $i \geq 1$ and $r_{j} \leq p+q$ for $j \neq t_{i}, s_{j} \leq p+q$ for $j \neq u_{i}$. Suppose the result is shown for $1+R_{i}$ with $g \leq i<t_{k}$ and $1+S_{i}$ with $h \leq i<u_{l}$. For $k=l=1$ this follows from the definitions of $\lambda, \mu, \tilde{g}, \tilde{h}, t$ and $u$. We have either $r_{t_{k}}<1+S_{u_{t}}$ or $s_{u_{l}}<1+R_{t_{k}}$, because otherwise $r_{t_{k}}=s_{u_{l}}=\infty$ by lemma 5. Suppose $r_{t_{k}}<1+S_{u_{i}}$. We show that

$$
\begin{array}{ll}
R_{t_{k}+j}-R_{t_{k}-1}=v p+w q \quad \text { with }(v, w) \in K \cup K_{1} \cup K_{\mu} \text { if } j=0 \\
& \text { and }(v, w) \in K \cup K_{1} \text { if } 0<j<t_{k+1}-t_{k} .
\end{array}
$$

This implies (i) for $1+R_{i}$ with $t_{k} \leq i<t_{k+1}$, since $1+R_{t_{k}+j}=1+R_{t_{k}-1}+R_{t_{k}+j}-R_{t_{k}-1}$ and $1+R_{t_{k}-1}=v p+w q$ with $(v, w) \in K \cup K_{\lambda}$ by the induction hypothesis. It also implies the second assertion of (i). If $\mu=1$, we get from (3.21) and from $(v, w) \in K_{1}$ for some $j$, that $(v, w) \in K_{1}$ for $j=0$, since $r_{t_{k}+j}$ for $1 \leq j<t_{k+1}-t_{k}$ is either $p$ or $p+q$, i.e. $R_{t_{k}+j}-R_{t_{k}}=v^{\prime} p+w^{\prime} q$ with $w^{\prime} / v^{\prime} \leq 1$. 
The proof is finished once we have shown (3.21). By lemma $5, r_{t_{k}}=1+S_{i}$ with $h<i<u_{l}$, hence for $j=0(3.21)$ is satisfied by the induction hypothesis. Furthermore $r_{t_{k}}-s_{i}=1+S_{i-1}$ and $h \leq i-1<u_{l}$, hence, again by the induction hypothesis,

$$
r_{t_{k}}-s_{i}=v p+w q \quad \text { with }(v, w) \in K \cup K_{1} \cup K_{\mu} .
$$

By lemma $5, s_{i}=1+R_{Q(i)}$, and by lemma 6 we have an $m \geq 1$ with $r_{t_{k}+j}=r_{Q^{(i)+j}}$ for $1 \leq j<m$ and $r_{t_{k}+m}>r_{Q(i)+m}$. As

$$
R_{t_{k}+j}-R_{t_{k}-1}=r_{t_{k}}-s_{i}+1+R_{Q(i)+j}
$$

we get (3.21) for $1 \leq j<m$ by (3.22) and induction, since $g-1 \leq Q(i)<t_{k}$, by definition of $Q(i),(3.4)$ and the irreducibility of $\mathscr{D}^{\prime}$. If $t_{k}+m \geq t_{k+1}$, the induction step is finished. Otherwise $r_{t_{k}+m} \leq p+q$, and we get from $r_{t_{k}+m}>r_{Q(i)+m}$, that

$$
r_{t_{k}+m}=p+q \text { and } r_{Q(i)+m}=p \text {. }
$$

Hence it follows by induction as above that

$$
R_{t_{k}+m-1}-R_{t_{k}-1}+p=v p+w q \quad \text { with }(v, w) \in K \cup K_{1} .
$$

We remark that $r_{t_{k}+j}$ is $p$ or $p+q$ for $1 \leq j<t_{k+1}-t_{k}$, hence $\underline{d}_{l_{0}+j}$ is defined for $l_{0}=t_{k}+m-g+1$ and $0 \leq j<t_{k+1}-t_{k}-m$. Now define $l_{1}, l_{2}, \ldots$ inductively, such that $\underline{d}_{l_{0}+l_{1}+\cdots+l_{n}+j}=\underline{d}_{j}$ for $1 \leq j<l_{n+1}$ and $\underline{d}_{l_{0}+\cdots+l_{n+1}}=(1,1)>\underline{d}_{l_{n+1}}=(1,0)$ (cf. lemma 7). For $0 \leq j<l_{n+1}$ we have then

$$
R_{t_{k}+m+l_{1}+\cdots+l_{n}+j}-R_{t_{k}+m-1}=v p+w q
$$

with

$$
\begin{aligned}
(v, w) & =(1,1)+\underline{d}_{1}+\cdots+\underline{d}_{l_{1}-1}+\cdots+(1,1)+\underline{d}_{1}+\cdots+\underline{d}_{j} \\
& =(1,0)+\underline{c}_{l_{1}}+\cdots+\underline{c}_{l_{n}}+\underline{c}_{j},
\end{aligned}
$$

since $\underline{d}_{b_{0}}=(1,1)$ by $(3.23), \underline{d}_{l_{i}}=(1,0)$ and $\underline{d}_{l_{0}+\cdots+l_{i}}=(1,1)$ for $i \geq 1$, and $\underline{c}_{0}=(0,1)$. Together with (3.24), from this we get (3.21) for $m \leq j<t_{k+1}-t_{k}$, as $\underline{c}_{l_{i}} \in K \cup K_{1} \cup K_{\lambda}$ by induction. This completes the proof.

LEMMA 12. If $\lambda=1$ then $1+R_{g+i}=v p+w q$ with $(v, w) \in K$ for $i \geq 1$ or there are $k \geq 1$ and $z \geq 2$ such that $1+R_{g+i}=v p+w q$ with $(v, w) \in K$ for $1 \leq i<k$ and $(v, w)=(z, z) \in$ $K_{1}$ for $i=k$. Then $j(p+q) \in Z\left(\mathscr{D}^{\prime}\right)$ for $j \geq z$.

Proof. Suppose that there is a $k \geq 1$ with $1+R_{g+k}=v p+w q$ with $(v, w) \in K_{1}$. Otherwise we get $(v, w) \in K$ for all $k \geq 1$ by lemma 11 . If $k=1$ then the requirements of lemma 10 are satisfied (cf. lemma 11) and the desired result follows. Otherwise let $k>1$ be minimal. By lemma 11 , we have $1+R_{k-1}=v p+w q$ with $(v, w) \in K$ and $r_{k}=1+S_{P(k)}=v p+w q$ with $(v, w) \in K \cup K_{1} \cup K_{\mu}$, if $r_{k} \geq p+q$. This gives $1+R_{k}=$ $v p+w q$ with $(v, w) \in K$, a contradiction, hence $r_{k}=p$. But then we have a closed path of length $1+R_{k}=z p+z q$, which is not disjoint from the closed path given by $(g, h)$. By (3.10) the desired result follows.

A similar lemma holds for $S_{h+i}$ instead of $R_{g+i}$. We denote the number corresponding to $z$ by $z^{\prime}$.

LEMMA 13. (i) If $r_{i+1}=\infty$ for some $i$ and $s_{j}<\infty$ for all $j$, then $Z(\overline{\mathscr{D}})=$ $Z\left(\overline{\mathscr{D}} \backslash\left\{A_{k}: k>R_{i}\right\}\right)$. 
(ii) If $r_{i+1}=\infty$ and $s_{j+1}=\infty$, then $Z(\overline{\mathscr{D}})=\left\{R_{i}+S_{j}+2\right\} \cup Z\left(\overline{\mathscr{D}} \backslash\left\{A_{k}, B_{l}: k>R_{i}\right.\right.$, $\left.1>S_{j}\right\}$ ).

Proof. (i) It follows from (3.1) that $r_{i+1}=\infty$ implies $\sigma^{m} \underline{a}=\underline{b}$, where $m=1+R_{i}$. If one cancels $\left\{A_{k}: k \geq m\right\}$ from $\overline{\mathscr{D}}$, one loses a representation of $\sigma^{m} \underline{a}=\underline{b}$. But $\underline{b}$ is also represented by the path $A_{q} \rightarrow A_{q+1} \rightarrow \cdots \rightarrow A_{q+p-1} \rightarrow B_{p} \rightarrow B_{p+1} \rightarrow \cdots$ in $\overline{\mathscr{D}} \backslash\left\{A_{k}: k>R_{i}\right\}$.

(ii) If one cancels $\left\{A_{k}, B_{l}: k>R_{i}, l>S_{j}\right\}$ from $\mathscr{D}^{\prime}$, one loses the closed path given by (3.7). It has length $1+R_{i}+1+S_{j}$.

LEMMA 14. Suppose that (3.6) occurs. Then we have a closed path $\mathscr{G}=$ $\left(A_{k}: R_{i}<k \leq R_{i+j}\right)$ of length $U=R_{i+j}-R_{i}$. Suppose there is no other closed path of length $U$ in $\mathscr{D}^{\prime}$. Then $U=v p+w q$ with $f:=(v, w) \in K_{\lambda}$ and one of the following holds:

(i) $t=\infty$ and there is a $\underline{c}_{n} \in K_{\lambda}$ such that $\mathscr{G}$ is not disjoint from $\mathscr{R}_{n}$;

(ii) $t=\infty$ and the entries of $f$ have no common divisor;

(iii) $\lambda=1, i=g, z$ of lemma 12 exists and $f=(z-1, z-1)$.

Proof. It follows from (3.1) and (3.6) that

$$
r_{i+m}=r_{i+j+m} \quad \text { and } \quad U=R_{i+j+m}-R_{i+m} \quad \text { for } m \geq 1 .
$$

If $\underline{f} \in K$, then there is another closed path of length $U$ in $\mathscr{D}^{\prime}$ by proposition 2 . Hence we suppose

$$
\underline{f} \notin K
$$

We consider first the case that

$$
r_{i+k}>p+q \quad \text { for some } k \geq 1 \text {, i.e. } r_{i+k}=1+S_{m} \text { with } m>h \text {. }
$$

If $\lambda<1$ it follows from lemma 11 and (3.25) that $\underline{f} \in K \cup K_{\mu}$. By (3.26) we have $\underline{f} \in K_{\mu}$. Again by lemma 11, we get $U=1+S_{m}$, i.e. $\underline{f}=\underline{c}_{m-g+1}^{\prime}$, if $\mu>1$ (cf. (ii) of lemma 11), hence we have another closed path of length $U$ in $\mathscr{D}^{\prime}$. If $\mu=1$ then

$$
U=n(p+q) \geq 1+S_{m}=n^{\prime}(p+q)
$$

(cf. the last statement of (i) of lemma 11) and $n \geq n^{\prime} \geq z^{\prime}$ by lemma 12 . Hence there is another closed path of length $U$ in $\mathscr{D}^{\prime}$.

If $\lambda=1$ then $\mu>1$, i.e. (3.14) holds. By lemma 11 and (3.26), we get $f \in K_{1} \cup K_{\mu}$. If $f \in K_{\mu}$ the same argument as above applies. Hence we suppose $f \in K_{1}$, i.e. $U \stackrel{=}{=} n(p+q)$ for some $n$. If $m$ defined in (3.27) satisfies $m<\tilde{h}$, then $s_{m}=p+q$ by (3.14) and $r_{k+i+1} \geq r_{g+1}$ by lemma 6 . If $r_{g+1}<1+S_{\tilde{h}}$, then

$$
1+R_{g+1}=p+q+r_{g+1}=l(p+q)
$$

with $l \geq z$ (cf. lemma 12) and $U \geq r_{\mathrm{g}+1} \geq(z-1)(p+q)$ by (3.25). Hence we have already $U \in Z\left(\mathscr{D}^{\prime}\right)$ or (iii) occurs by lemma 12 . If $r_{i+k+1} \geq 1+S_{\tilde{h}}$ we have the case we consider now, with $k+1$ instead of $k$. Suppose now $m$ of (3.27) satisfies $m \geq \tilde{h}$. It follows then from lemma 6 , that $m=\tilde{h}, r_{i+k+v}=p+q$ for $1 \leq v<l$ and $r_{i+k+l}=p$, for some $l$, otherwise $f \notin K_{1}$. But then $r_{g+1}=1+S_{\tilde{h}}, r_{g+1+v}=p+q$ for $1 \leq v<l^{\prime}$ and $r_{\mathrm{g}+1+l^{\prime}}=p$, for some $l^{\prime} \leq l$ by lemma 6 , which gives $1+R_{\mathrm{g}+1+l^{\prime}}=w(p+q)$ for some $w$ and $z \leq w$ (cf. lemma 12). We get again $U \geq R_{g+1+1}-R_{g} \geq(z-1)(p+q)$ and either $U \in Z\left(\mathscr{D}^{\prime}\right)$, by lemma 12 , or (iii) holds. 
Next we suppose that (3.27) does not hold, i.e.

$$
r_{i+k} \leq p+q \quad \text { for all } k \geq 1 \text {. }
$$

Then $\underline{d}_{m}$ is defined for $m \geq i-g+2$ and $\underline{f}=\underline{d}_{i-g+2}+\cdots+\underline{d}_{i-g+j+1}$ by (3.25). Clearly $\underline{f} \leq(1,1)$. We get that

$$
\underline{f} \in K \cup K_{\lambda} \cup K_{\mu}
$$

as $1+R_{i+k j}=1+R_{i}+k(v p+w q)$ with $(v, w)=\underline{f}, \underline{f} \notin K \cup K_{\lambda} \cup K_{\mu}$ would imply $1+$ $R_{i+k j}=v^{\prime} p+w^{\prime} q$ with $\left(v^{\prime}, w^{\prime}\right) \notin K \cup K_{\lambda} \cup K_{\mu}$, for large $k$, a contradiction to lemma 11. In addition to (3.28) we suppose for some $l$

$$
r_{l+k}=r_{g+k} \quad \text { for } k \geq 0 \text {, i.e. } i=g-1 \text {. }
$$

From this and (3.28), it follows that $t=\infty$. By (3.26), (3.29) and $f \leq(1,1)$, we have either $\underline{f}=(1,1)$ or $\underline{f} \in K_{\lambda}$. For $f=(1,1)$ we have $U=p+q \in Z\left(\overline{\mathscr{D}}^{\prime}\right)$, hence suppose $\underline{f} \in K_{\lambda}$. If there is an $\underline{e}_{m}=\underline{c}_{n} \in \bar{K}_{\lambda}$, then $\underline{d}_{n}=(1,0)$ by proposition 1 and the path $\mathscr{R}_{n}$ is not disjoint from $\mathscr{G}$ by (3.30). This is (i). If $\underline{e}_{m} \notin K_{\lambda}$ for all $m$, then for every minimal $\underline{c}_{n}=\underline{e}_{m}$ we have $\underline{c}_{n}+f=\underline{c}_{n+j}<\underline{c}_{n}$, so that $\underline{e}_{m+1}=\underline{c}_{n}$, with $n^{\prime} \leq n+j$. Then there is a $k$ such that $m_{i}=2$ for $i \geq k$ (cf. proposition 1), because otherwise the gaps between the minimal $\underline{c}_{n}$ are not bounded by (3.20). By proposition 1, $\underline{e}_{k+v}=$ $\underline{e}_{k}+v\left(\underline{e}_{k+1}-\underline{e}_{k}\right)$ for $v \geq 1$, and $\underline{e}_{k+1}-\underline{e}_{k} \in K_{\lambda}$, since the slope of $\underline{e}_{k+v}$ decreases to $\lambda$. The sequence $\left(\underline{d}_{n}\right)$ has period $j$ and $\underline{c}_{n+j}-\underline{c}_{n}=\underline{e}_{k+1}-\underline{e}_{k}$, so that $\underline{f}=\underline{e}_{k+1}-\underline{e}_{k}$. By proposition 1, the entries of $\underline{e}_{k+1}-\underline{e}_{k}$ have no common divisor (cf. the proof of proposition 2) and (ii) occurs.

Now suppose that (3.28) holds and (3.30) does not hold. For $m=i-g+1$ define $l_{1}, l_{2}, \ldots$ inductively by

$$
\begin{aligned}
& \underline{d}_{m+l_{1}+\cdots+l_{k}+n}=\underline{d}_{n} \quad \text { for } 1 \leq n<l_{k+1}, \\
& \underline{d}_{m+l_{1}+\cdots+l_{k}+l_{k+1}}=(1,1)>\underline{d}_{l_{k+1}}=(1,0) .
\end{aligned}
$$

These vectors are defined by (3.28) (cf. lemmas 5 and 7). Because (3.30) does not hold, $l_{k}<\infty$ for all $k$. By (3.25) we have $f=\underline{d}_{m}+\cdots+\underline{d}_{m+j-1}$. Let $a \geq 1$ be such that $l_{1}+\cdots+l_{a-1}<j \leq l_{1}+\cdots+l_{a}$. We show that

$$
l_{1}+\cdots+l_{a}-j=l_{1}+\cdots+l_{b} \quad \text { for some } b \text { with } 0 \leq b<a .
$$

Set $V=l_{b+1}+\cdots+l_{a}-j$ and suppose $0 \leq V<l_{b+1}$. We have to show $V=0$. By (3.25) we have

$$
\underline{d}_{m+k}=\underline{d}_{m+j+k} \quad \text { for } k \geq 1
$$

Hence it follows from (3.31) that

$$
\underline{d}_{m+j+l_{1}+\cdots+l_{b}+k}=\underline{d}_{k} \quad \text { for } 1 \leq k<l_{b+1} .
$$

Again by (3.31) for $k=a-1$ and $n=n^{\prime}+k$, where $n^{\prime}=j-l_{b+1}-\cdots-l_{a-1}$, we get

$$
\begin{aligned}
& \underline{d}_{m+j+l_{1}+\cdots+l_{b}+k}=\underline{d}_{n^{\prime}+k} \quad \text { for } 1 \leq k<V, \\
& \underline{d}_{m+j+l_{1}+\cdots+l_{b}+v}>\underline{d}_{n^{\prime}+v} .
\end{aligned}
$$

As $V<l_{b+1}$, we get from (3.34) and (3.35) that $\underline{d}_{n^{\prime}+k}=\underline{d}_{k}$ for $1 \leq k<V$ and $\underline{d}_{n^{\prime}+V}<\underline{d}_{V}$, a contradiction to lemma 7, unless $V=0$. Hence (3.32) is shown.

Set $m^{\prime}=m+l_{1}+\cdots+l_{b}$. By (3.33) and (3.32) we get

$$
\underline{f}=\underline{d}_{m^{\prime}}+\underline{d}_{m^{\prime}+1}+\cdots+\underline{d}_{m^{\prime}+l_{b+1}+\cdots+l_{a}-1} \text {. }
$$


By (3.31) we get $\underline{f}=(1,1)+\underline{c}_{l_{b+1}-1}-\underline{c}_{0}+\cdots+(1,1)+\underline{c}_{l_{a}-1}-\underline{c}_{0}$, which is $\underline{c}_{l_{b+1}}+\cdots+$ $\underline{c}_{l_{a}}$, since $\underline{d}_{l_{k}}=(1,0)$. As $\underline{d}_{l_{k}}=(1,0)$ the closed paths $\mathscr{R}_{l_{k}}$ of lengths $v p+w q$ with $(v, w)=c_{l_{k}}$ are not disjoint, so that we have a closed path of length $U$ in $\mathscr{D}^{\prime}$ by (3.10.). This finishes the proof.

If the second assertion of (3.6) is valid, a lemma similar to lemma 14 holds. Now we can show:

Proposition 3. $Z(\overline{\mathscr{D}}) \subset\left\{v p+w q:(v, w) \in K \cup K_{\lambda} \cup K_{\mu}\right\}$ and $Z(\overline{\mathscr{D}})=Z\left(\mathscr{D}^{\prime}\right)$.

Proof. Let $\mathscr{P}$ be a closed path in $\overline{\mathscr{D}}$ and denote its length by $L$. We have to show

$$
\begin{array}{ll}
L=v p+w q & \text { with }(v, w) \in K \cup K_{\lambda} \cup K_{\mu} ; \\
L=v p+w q & \text { with }(v, w) \in K_{\lambda} \Rightarrow L \in Z\left(\mathscr{D}^{\prime}\right) .
\end{array}
$$

Then a result analogous to (3.37) also holds for $(v, w) \in K_{\mu}$ and $Z\left(\mathscr{D}^{\prime}\right)=Z(\overline{\mathscr{D}})$ follows from proposition 2 .

If $\mathscr{P}$ does not contain an $A_{i}$ and a $B_{j}$ then (3.6) holds and $\mathscr{P}$ is the path $\mathscr{G}$ of lemma 14 , or one which contains only $B_{j}$. Then the case considered below and lemma 14 imply (3.36) and (3.37). If (i) or (ii) holds, then $t=\infty$ implies $\mathscr{G} \subset \mathscr{D}^{\prime}$ which gives (3.37). If (iii) holds, this follows from $i=g$ and $(v, w) \in K_{1}$.

Now we suppose that $A_{i}, B_{j} \in \mathscr{P}$ for some $i, j$. We divide $\mathscr{P}$ into segments $A_{T} \rightarrow A_{T+1} \rightarrow \cdots \rightarrow A_{U}$ and $B_{V} \rightarrow B_{V+1} \rightarrow \cdots \rightarrow B_{W}$. Because $B_{k} \rightarrow A_{T}$ for some $k$ and $A_{U} \rightarrow B_{l}$ for some $l$, we have $T=s_{i}$ for some $i$ and $U=R_{j}$ for some $j$ by (3.4). Similar results hold for $V$ and $W$. Hence there is an $n$ and $k_{i} \geq g, l_{i} \geq h$ with $l_{n}=l_{0}$ such that

Hence

$$
\mathscr{P}=\bigcup_{i=1}^{n}\left\{A_{i}, B_{w}: s_{l_{i-1}} \leq v \leq R_{k_{i}}, r_{k_{i}} \leq w \leq S_{l_{i}}\right\}
$$

$$
L=\sum_{i=1}^{n}\left(R_{k_{i}}-s_{l_{i-1}}+1\right)+\left(S_{l_{i}}-r_{k_{i}}+1\right)=\sum_{i=1}^{n}\left(R_{k_{i}-1}+1\right)+\left(S_{l_{i}-1}+1\right) .
$$

If $k_{i}=g$, then $s_{l_{i-1}}=q=R_{k_{i}-1}+1$, since $s_{l_{i-1}} \leq R_{k_{i}}$, hence $R_{k_{i}-1}+1+S_{l_{i-1}-1}+1=$ $S_{l_{i-1}}+1$. A similar result holds if $l_{i}=h$. Hence we get (cf. also lemma 13):

$L$ is a sum of $1+R_{i}$ and $1+S_{j}$ with $i \geq g$ and $j \geq h$.

Now (3.36) follows from lemma 11. If $\lambda>1$ (3.37) also follows, since then there are only $1+R_{i}=v p+w q$ with $(v, w)=c_{m} \in K_{\lambda}$ in (3.39). This gives $\underline{d}_{m}=(1,0)$, because $\underline{d}_{m}=(1,1)$ implies that $\underline{c}_{m-1}$ has a slope smaller than $\lambda$. Then $\mathscr{P}$ is a union of paths $\mathscr{R}_{n} \subset \mathscr{D}^{\prime}$. If $\lambda=1$ then either $L=p+q$, or (3.39) contains an $1+R_{i}=v p+w q$ with $i>g$ and $(v, w) \in K_{1}$. By lemma 12 , we get $v=w \geq z$ and $L=m(p+q)$ with $m \geq z$, i.e. $L \in Z\left(\mathscr{D}^{\prime}\right)$. This shows (3.37) and finishes the proof.

Recall that the set $F$ of all $\underline{x}$ represented as one-sided paths in $\overline{\mathscr{D}}$ is $\sigma$-invariant and a finite union of intervals, which contains $\Omega$ (cf. [6]). Hence $Z(F)=Z(\overline{\mathscr{D}})$ (cf. $\S 1)$.

THEOREM 2. Let $\Omega$ be a topologically transitive subset of $\Sigma_{T}^{+}$. Then there are integers $p$ and $q$ and real numbers $\lambda$ and $\mu$, such that

$$
\{v p+w q: \lambda<w / v<\mu\} \subset Z(\Omega) \subset\{v p+w q: \lambda \leq w / v \leq \mu\} .
$$


For $\alpha=\lambda$ or $\mu$ we have $Z(\Omega) \cap\{v p+w q: w / v=\alpha\}$ is empty, or $\{k p+l q\}$ or $\{m(k p+$ $l q): m=1$ or $m \geq z\}$ for some $z \geq 2$, where the integers $k$ and $l$ have no common divisor and satisfy $l / k=\alpha$.

If $\Omega$ does not consist of a periodic orbit only, we have $Z(F)=Z(\Omega)$ and either $\lambda<1 \leq \mu$ or $\lambda \leq 1<\mu$.

Proof. For the cases considered at the beginning of $\S 3$, the theorem is easily checked. Hence suppose the converse of (3.12). Since $Z\left(\mathscr{D}^{\prime}\right) \subset Z(\Omega)$ by lemma 2 , it follows from $Z(\bar{D})=Z(F)$ and proposition 3 that $Z\left(\mathscr{D}^{\prime}\right)=Z(\Omega)$ and $Z(\Omega)=Z(F)$. Since either (3.13) or (3.14) holds, we have $\lambda<1$ or $\mu>1$. The first result of the theorem follows from propositions 2 and 3. The second result for $\alpha=\lambda$ follows for $\lambda=1$ from lemmas 12 and 14 and for $\lambda>1$ either from lemma 14 or by the fact that the entries of a minimal $\underline{c}_{i}$ have no common divisor (cf. the proof of proposition 2) and the fact that $\underline{c}_{m}, \underline{c}_{n} \in K_{\lambda}$ implies $\underline{d}_{m}=\underline{d}_{n}=(1,0)$ and hence the paths $\mathscr{R}_{m}$ and $\mathscr{R}_{n}$ are not disjoint (use (3.10)).

Now we look for monotonic mod 1 transformations $T$, which have a given set of integers as the set $Z(T)=\{n$ : there is an $x$ which has period $n$ under $T\}$.

LEMMA 15. Let $T$ on $[0,1)$ be monotonic mod 1 with $N=2$ (for the definition of $N$ see the beginning of $\S 3$ ). For every integer $n$ there is a monotonic mod 1 transformation $S$ on $[0,1)$ with $Z(S)=\{n\} \cup\{n k: k \in Z(T)\}$. If $n \geq 2$ then $S$ has $N=2$.

Proof. Set $a=T(0)$ and $b=\lim _{t \uparrow 1} T(t)$. We consider first the case $n \geq 2$ and define $S$ on $I=[-1-b, 2 n-1)$ instead of $[0,1)$. We consider $[0,1)=J_{1} \cup J_{2}$, on which $T$ is defined, as a subset of $I$. Set $K_{-2}=[-1-b,-1), K_{i}=[i, i+1)$ for $-1 \leq i \leq 2 n-4$, $K_{2 n-3}=[2 n-3,2 n-2+a)$ and $K_{2 n-2}=[2 n-2+a, 2 n-1)$. Now we define $S$ on $I$ by

$$
S(x)= \begin{cases}x+2 n-3+b & \text { for } x \in K_{-2}, \\ (2+a-b) x+2 n-2+a & \text { for } x \in K_{-1}, \\ T(x)+2 n-2 & \text { for } x \in J_{1} \subset K_{0}, \\ T(x)-1-b & \text { for } x \in J_{2} \subset K_{0}, \\ x-2 & \text { for } x \in[1,2 n-1)=\bigcup_{i=1}^{2 n-2} K_{i} .\end{cases}
$$

One checks that $S$ is continuous at $x=-1, x=0$ and $x=1$, so that $S$ is monotonic $\bmod 1$ with $N=2$. Furthermore $S\left(J_{1}\right)=K_{2 n-2}, S\left(J_{2}\right)=K_{-2}, S\left(K_{2 n-2}\right) \subset K_{2 n-4}$, $S\left(K_{-2}\right) \subset K_{2 n-4}$ and $S\left(K_{2 i}\right)=K_{2 i-2}$ for $n-2 \geq i \geq 1$. Since $S$ has slope 1 on $I \backslash[-1,1)$, we get that $S^{n} \mid[0,1)=T$. We have also $S\left(K_{2 i+1}\right) \supset K_{2 i-1}$ for $n-2 \geq i \geq 0$ and $S\left(K_{-1}\right) \supset K_{2 n-3}$. This gives the desired result about $Z(S)$.

For $n=1$ we consider $[0,2)$ instead of $[0,1)$. We define $f:[0,1) \rightarrow[0,2)$ by $f(x)=$ $x-c$ for $x \in J_{2}=[c, 1)$ and $f(x)=x-c+2$ for $x \in J_{1}=[0, c)$. Set $S(x)=f\left(T\left(f^{-1}(x)\right)\right)$ for $x \in[0,1-c) \cup[2-c, 2)=f([0,1))$. Then we have $S(2-c)=f(a)$ and $\lim _{t \uparrow 1-c} S(t)=f(b)$. If $a \in J_{1}$ and $b \in J_{2}$, then $f(a)>f(b)$ and we define $S$ to be linear on $[1-c, 2-c]$ with $S(1-c)=f(b)$ and $S(2-c)=f(a)$. If both $a$ and $b$ are in $J_{1}$ or in $J_{2}$, we define $S$ to be linear on $[1-c, 1)$ and on $[1,2-c]$ with $S(1-c)=f(b)$, $\lim _{t \uparrow 1} S(t)=2, S(1)=0$ and $S(2-c)=f(a)$. If $a \in J_{2}$ and $b \in J_{1}$, we introduce in the same way two discontinuities at $x, y \in(1-c, 2-c)$ such that $S([x, y))=[0,2)$, 
$S(y)=0$ and $\lim _{t \uparrow x} S(t)=2$. In any case $S$ is monotonic $\bmod 1$ with $N=3$ and $S$ has no periodic point in $[1-c, 2-c)$ except one fixed point.

Proposition 4. Let $p, q \in \mathbb{N}, \lambda \in[0,1]$ and $\mu \in(1, \infty]$ be given, such that $p$ and $q$ have no common divisor. Then there is a monotonic mod 1 transformation $T$ on $[0,1)$ with $N=2$ such that

$$
\{v p+w q: \lambda<w / v<\mu\} \subset Z(T) \subset\{v p+w q: \lambda \leq w / v \leq \mu\} .
$$

Furthermore $T$ is topologically transitive.

Proof. The Markov diagram $\mathscr{D}$ of $T$ is determined by sequences $r_{1}, r_{2}, \ldots$ and $s_{1}, s_{2}, \ldots$ of integers (cf. the beginning of $\S 3$ ). We shall construct such sequences, so that $Z(T)=Z(\mathscr{D})$ has the desired property, and then a monotonic mod 1 transformation $T$ giving rise to these $r_{i}$ and $s_{i}$.

Suppose $p \geq q$ and set $p_{0}=p$ and $q_{0}=q$. Then there are uniquely determined $k_{i} \geq 1, l_{i} \geq 1, p_{i}$ and $q_{i}$ for $i \geq 0$ such that

$$
\begin{aligned}
& p_{i}=k_{i} q_{i}+p_{i+1} \quad \text { with } 0 \leq p_{i+1}<q_{i}, \\
& q_{i}=l_{i} p_{i+1}+q_{i+1} \quad \text { with } 0 \leq q_{i+1}<p_{i+1} .
\end{aligned}
$$

As $p$ and $q$ have no common divisor, we end with $p_{m}=1$ or $q_{m}=1$ for some $m$. Suppose $p_{m}=1$. We define (if $q_{m}=1$ one has to begin with $s_{i}$ )

$$
\begin{array}{ll}
r_{i}=1=p_{m} & \text { for } 1 \leq i<q_{m-1}=l_{m-1}, \\
s_{i}=q_{m-1} & \text { for } 1 \leq i \leq k_{m-1} .
\end{array}
$$

We set $R_{i}=r_{1}+r_{2}+\cdots+r_{i}, S_{i}=s_{1}+s_{2}+\cdots+s_{i}$ for $i \geq 1$ and $L_{i}=l_{i}+l_{i+1}+\cdots+l_{m-1}$, $K_{i}=k_{i}+k_{i+1}+\cdots+k_{m-1}$ for $0 \leq i \leq m-1$. For $j=m-2, m-3, \ldots, 1,0$ define

$$
\begin{array}{ll}
r_{i}=1+S_{K_{j+1}} & \text { for } L_{j+1} \leq i<L_{j}, \\
s_{i}=1+R_{L_{j}-1} & \text { for } K_{j+1}<i \leq K_{j} .
\end{array}
$$

From (3.41) we get by induction that $1+S_{K_{i+1}}=p_{j+1}$ and $1+R_{L_{i}-1}=q_{j}$. Finally we define

$$
\begin{array}{ll}
r_{g}=1+S_{K_{0}}=p & \text { where } g=L_{0}, \\
s_{h}=1+R_{L_{0}-1}=q & \text { where } h=K_{0}+1 .
\end{array}
$$

Then the Markov diagram restricted to $\left\{A_{i}, B_{i}: 1 \leq i \leq p+q\right\}$, which is determined by $r_{i}$ for $i \leq g$ and by $s_{i}$ for $i \leq h$, contains only the closed path (3.11) (cf. also [4] and [6]).

The $r_{i}$ for $i>g$ are determined by $\lambda$, the $s_{i}$ for $i>h$ are determined by $\mu$. We consider only the $r_{i}$. If $\lambda=1$ we set $r_{i}=p+q$ for all $i \geq g$. Now suppose $\lambda \in[0,1)$. According to proposition 1 , set $\underline{e}_{0}=(0,1)$ and $\underline{e}_{1}=(1,1)$. If $\underline{e}_{i-1}$ and $\underline{e}_{i}$ are defined, such that $\underline{e}_{i}$ has slope $>\lambda$ and $\underline{e}_{i}-\underline{e}_{i-1}$ has slope $<\lambda$, then define

$$
m_{i}=\min \left\{m \geq 1: \text { slope of } m \underline{e}_{i}-\underline{e}_{i-1} \geq \lambda\right\},
$$

which exists since $\underline{e}_{i}$ has slope $>\lambda$. We have $m_{i} \geq 2$, since $\underline{e}_{i}-\underline{e}_{i-1}$ has slope $<\lambda$. Set $\underline{e}_{i+1}=m_{i} \underline{e}_{i}-\underline{e}_{i-1}$. If the slope of $\underline{e}_{i+1}$ equals $\lambda$, we stop the definition of $\underline{e}_{j}$ 's. Otherwise $\underline{e}_{i+1}$ has slope $>\lambda$ and $\underline{e}_{i+1}-\underline{e}_{i}=\left(m_{i}-1\right) \underline{e}_{i}-\underline{e}_{i-1}$ has slope $<\lambda$, so that we can continue the definition of $e_{j}$ 's. If there are infinitely many $\underline{e}_{j}$ 's, then the slope of $\underline{e}_{j}$ decreases to $\lambda$, if $j \rightarrow \infty$. 
Now we define the $r_{i}$ for $i>g$. Set $t_{0}=0, t_{1}=1$ and $t_{i+1}=t_{i} m_{i}-t_{i-1}$ for $i \geq 1$. Then set for $i \geq 1$

$$
\begin{array}{cll}
r_{g+t_{i} k}=p+q, \quad r_{g+t_{i} k+j}=r_{g+j} \quad\left(1 \leq j<t_{i}\right) & \text { for } 1 \leq k \leq m_{i}-2, \\
r_{g+t_{i} k+j}=r_{g+t_{i-1}+j} \quad\left(0 \leq j<t_{i}-t_{i-1}\right) & \text { for } k=m_{i}-1
\end{array}
$$

If there are infinitely many $\underline{e}_{j}$ we have defined all $r_{i}$. If $\underline{e}_{n}$ is the last $\underline{e}_{j}$ which is defined, we have defined $r_{i}$ for $i<g+t_{n+1}$. We set $r_{i}=p+q$ for $i \geq g+t_{n+1}$. In the same way as for the $r_{i}$ one defines the $s_{i}$ using $\mu$ instead of $\lambda$.

Next we define $\underline{a}$ and $\underline{b} \in\{1,2\}^{\mathbb{N}}$. Set $a_{R_{k}}=1$ and $b_{S_{k}}=2$ for $k \geq 0$. Then one can define $\underline{a}$ and $\underline{b}$ inductively from the $r_{i}$ 's and $s_{i}$ 's using (3.1) and (3.2). Then $\underline{a}$ and $\underline{b}$ give rise to a Markov diagram determined by these $r_{i}$ and $s_{i}$. The proof of proposition 1 shows that these $r_{i}$ and $s_{i}$ give rise to the $\underline{e}_{i}$ 's constructed above, so that, by propositions 2 and $3, Z(\mathscr{D})$ has the properties we require from $Z(T)$. Hence the proof is finished if we find a $T$ on $[0,1)$ which is monotonic mod 1 with $N=2$ and which satisfies

$$
\varphi(0)=\underline{a} \quad \text { and } \quad \lim _{t \uparrow 1} \varphi(t)=\underline{b} .
$$

By results of [6], such a $T$ is topologically transitive.

It follows from the definitions that the $r_{i}$ and $s_{i}$ above satisfy the assertions of lemmas 5 and 6. these lemmas are proved in [3] and [4] using only the fact that

$$
\underline{a} \leq \sigma^{i} \underline{a} \leq \underline{b}, \quad \underline{a} \leq \sigma^{i} \underline{b} \leq \underline{b} \quad \text { for } i \geq 1,
$$

where $\leq$ denotes the lexicographic ordering in $\{1,2\}^{\mathbb{N}}$. These proofs even show that the assertions of lemmas 5 and 6 are equivalent to $(3.43)$, so that $(3.43)$ holds for $\underline{a}$ and $\underline{b}$ defined above.

Now choose $a_{i}, b_{i} \in[0,1]$ with $a_{0}=0, b_{0}=1$ such that the $a_{i}$ and $b_{i}$ are in the same order as the $\sigma^{i} \underline{a}$ and $\sigma^{i} \underline{b}$, where $a_{i}$ corresponds to $\sigma^{i} \underline{a}$ and $b_{i}$ corresponds to $\sigma^{i} \underline{b}$. This is possible by (3.43). Furthermore choose $c \in(0,1)$ such that $a_{i} \in[0, c]$ if $\sigma^{i} \underline{a} \in[1]$, and $a_{i} \in[c, 1]$ if $\sigma^{i} \underline{a} \in[2]$, and that $b_{i}$ and $\sigma^{i} \underline{b}$ have the same property. Define the graph of a transformation $T_{j}$, connecting the points $\left(c, a_{0}\right),\left(c, b_{0}\right)$ and $\left(a_{i}, a_{i+1}\right),\left(b_{i}, b_{i+1}\right)$ for $0 \leq i \leq j$ in $[0,1) \times[0,1)$ with straight lines. Since $\sigma$ is increasing on [1] and [2], these graphs $T_{j}$ converge for $j \rightarrow \infty$ to the graph of a transformation $T$, which is continuous and increasing on $[0, c)$ and on $[c, 1)$ and satisfies (3.42).

THEOREM 3. If $T$ is a monotonic mod 1 transformation then $Z(T)=\{n: \exists x \in[0,1]$ such that $x$ has period $n$ under $T\}$ satisfies one of the following, if it is not empty.

(i) $Z(T)=\left\{l_{i}: 1 \leq i<k\right\}$, where $k \leq \infty$ and $l_{i+1}>l_{i}$, such that $l_{i}$ divides $l_{i+1}$ for $i \geq 1$.

(ii) $Z(T)=\left\{l_{i}: 1 \leq i<k\right\} \cup \tilde{Z}(T)$, the $l_{i}$ as in (i) with $1 \leq k<\infty$ and there are $p, q \in \mathbb{N}, \lambda \in[0,1]$, and $\mu \in[1, \infty]$, such that

$$
\{v p+w q: \lambda<w / v<\mu\} \subset \tilde{Z}(T) \subset\{v p+w q: \lambda \leq w / v \leq \mu\},
$$

where the greatest common divisor of $p$ and $q$ is $l_{k-1}$ if $k>1$, and 1 if $k=1$, i.e. the set of $l_{i}$ 's is empty, and where either $\lambda<1$ or $\mu>1$. 
On the other hand, all sets $Z(T)$ described in (i) and for all $k, l_{i}, p, q, \lambda$ and $\mu$ as in (ii) a set $Z(T)$ with the properties of (ii) occurs for some monotonic mod 1 transformation $T$.

Proof. Let $\mathscr{D}_{1}, \mathscr{D}_{2}, \mathscr{D}_{3}, \ldots$ be the irreducible subsets of the Markov diagram $\mathscr{D}$ of $T$. There are finitely or countably many of them. They can be ordered such that there is a path from $\mathscr{D}_{i}$ to $\mathscr{D}_{i+1}$ (cf. [6]). Suppose $\mathscr{D}_{i}$ consists only of a closed path, which is then of the form (3.11). Denote $p$ and $q$ of (3.11) by $p_{i}$ and $q_{i}$. As described after lemma 6 , the irreducible subset $\mathscr{D}^{\prime}=\mathscr{D}_{i+1}$ contains the closed path (3.11). Now denote $p$ and $q$ of (3.11) by $p_{i+1}$ and $q_{i+1}$. It follows from $\S 2$ of [6], that the greatest common divisor of $p_{i+1}$ and $q_{i+1}$ is $l_{i}=p_{i}+q_{i}$, the length of the closed path $\mathscr{D}_{i}$. Hence, if all $\mathscr{D}_{i}$ consist only of closed paths, we get (i). If there is a $\mathscr{D}_{j}$ which does not consist of a closed path only, then we have (ii) by theorem 2 applied to $\mathscr{D}^{\prime}=\mathscr{D}_{j}$, where $\tilde{Z}(T)=Z\left(\overline{\mathscr{D}}_{\mathrm{j}}\right)=Z\left(\mathscr{D}_{\mathrm{j}}\right)$.

On the other hand, if we apply lemma $15 k-1$ times with $n=l_{k-1} / l_{k-2}, \ldots$, $n=l_{2} / l_{1}$ and $n=l_{1}$ to the transformation $T$ of proposition 4 , we get that all possible cases of (ii) occur for some monotonic mod 1 transformation $T$. If we do the same with $T(x)=x+a(\bmod 1)$ with irrational $a$, which has $Z(T)=\varnothing$, we get a $T_{k}$ with $Z\left(T_{k}\right)=\left\{l_{1}, l_{2}, \ldots, l_{k-1}\right\}$. The transformation $S$ defined by (3.40) depends only on $a=T(0)$ and $b=\lim _{\imath \uparrow 1} T(t)$ except for $x \in[0,1)$. If one renorms the interval $I$ in each step to $[0,1)$, this implies that $T_{k}$ converges to a monotonic mod 1 transformation $T$, which then satisfies $Z(T)=\left\{l_{i}: i \geq 1\right\}$.

\section{REFERENCES}

[1] L. Block, J. Guckenheimer, M. Misiurewicz \& L. S. Young. Periodic points and topological entropy of one-dimensional maps. Springer Lecture Notes in Mathematics 819, 18-34.

[2] F. Hofbauer. On intrinsic ergodicity of piecewise monotonic transformations with positive entropy. Israel J. Math. 34 (1979), 213-237. Part II, Israel J. Math. 38 (1981), 107-115.

[3] F. Hofbauer. Maximal measures for simple piecewise monotonic transformations. Zeitschr. Wahrsch. Verw. Gebiete 52 (1980), 289-300.

[4] F. Hofbauer. The maximal measure for linear mod one transformations. J. London Math. Soc. 23 (1981), 92-112.

[5] F. Hofbauer. The structure of piecewise monotonic transformations. Erg. Th. \& Dynam. Sys. 1 (1981), $159-178$.

[6] F. Hofbauer. Monotonic mod one transformations. Studia Math. To appear.

[7] L. Jonker \& D. Rand. Bifurcations in one dimension. Invent. Math. 62 (1981), 347-365.

[8] J. Milnor \& W. Thurston. On iterated maps of the interval. Preprint, Princeton 1977. 